\title{
Noisy Signaling: Theory and Experiment
}

\author{
Thomas de Haan Theo Offerman Randolph Sloof*
}

November 23, 2008

\begin{abstract}
We investigate a noisy signaling game, in which nature adds random noise to the (costly) message chosen. Theoretically, with an unfavorable prior the separating equilibrium vanishes for low noise. It reappears for intermediate levels of noise, where messages increase with noise. A pooling equilibrium always exists. In our experiment, noise works as an empirical equilibrium selection device. When noise increases, the separating equilibrium loses ground to the pooling equilibrium. Subjects separate for low noise where no separating equilibrium exists. Conditional on aiming for separation, high-quality senders choose messages that increase monotonically with noise. A simple behavioral explanation organizes the data well.
\end{abstract}

Keywords: signaling games, noise, separation, experiments

\section{Introduction}

Sometimes people choose very costly signals to deliver their messages in a credible way. For instance, during the 2005 Superbowl, firms paid 2.4 million dollars for broadcasting a 30-second spot. These costs came on top of the costs of producing the commercial, that amounted to an average of 350.000 dollars. ${ }^{1}$ If consumers infer the quality of a firm's product from its expenditure on advertising, highquality firms have an incentive to distinguish themselves from low-quality sellers by spending money on otherwise useless advertising (Nelson, 1970; 1974; Kihlstrom and Riordan, 1984; Milgrom and Roberts, 1986). As a somewhat more peculiar

\footnotetext{
*de Haan, Offerman, Sloof: Amsterdam School of Economics, Roetersstraat 11, 1018 WB Amsterdam, The Netherlands. We are grateful to CREED-programmer Jos Theelen for programming the experiment. The research of Theo Offerman has been made possible by a fellowship of the Royal Netherlands Academy of Arts and Sciences.

${ }^{1}$ These numbers are reported on http://www.gaebler.com/Television-Advertising-Costs.htm.
} 
example, the Yanomamö, a contemporary tribe of about twenty thousand Indians living in the Amazon rainforest on the border between Brazil and Venezuela, sometimes risk their lives in their vigorous pursuit of a fierce image (Chagnon, 1992). Disputes about women occasionally culminate in a club fight, where two males take turns striking each other on the head with a club of eight to ten feet long. The men are very proud of their heads that are covered with deep scars. Some men have a tonsure shaved on the top of their heads and they rub red pigment on their scars to make sure that nobody misses them. Regularly, people get killed in club fights or other outbursts of violence. Having a fierce image pays off among the Yanomamö. Chagnon (1988) reports that men who killed had on average two and half times as many wives and three times as many children than men who did not.

What these signaling examples have in common besides the high signaling costs, is that it is very hard for receivers to precisely judge the actual costs of the signal. A signal may be noisy because the production technology is noisy, as in the club fight example, where the deepness of a scar may be affected by incidental factors such as the angle in which the club hit the head. A signal may also be noisy because of imperfect knowledge of the receiver. This is likely to apply in the advertisement example, where most consumers only have an imprecise notion that the costs must be high. In fact, the assumption that there is no noise at all in the signal seems to be too strong in most real world applications. In this paper, we investigate theoretically and experimentally what happens when the assumption of no noise is dropped. Our results show that a noisy signaling game differs profoundly from a standard signaling game without noise, both in terms of theoretical predictions and in terms of experimental outcomes.

In his seminal paper, Spence (1973) provides the first game theoretic analysis of a signaling game in which a job applicant's investment in education does not improve her productivity. Like Spence, we focus on a pure signaling game in which signaling is completely wasteful. In our game a seller offers a product for sale that is either of high quality or of low quality. Nature first determines the quality of his product. ${ }^{2}$ Only the seller learns the actual quality. Then the seller chooses his level of signal costs, i.e. we equate the message he chooses with its costs. To these signal costs nature adds a random noise term. The buyer observes the resulting overall signal, but not the original signal costs, and decides whether or not to buy. Preferences are such that the buyer prefers to buy if and only if

\footnotetext{
${ }^{2}$ In our model we use the convention that sellers are males and buyers are females. In the experiment, of course, men and women were randomly assigned to the two roles.
} 
quality is high. Moreover, a sale is more valuable to a high-quality seller than to a low-quality seller. In this game signaling is completely wasteful, because both seller types would prefer to pool on zero signal costs if the buyer would ignore the seller's signal.

In our theoretical analysis we consider both the case of an unfavorable prior belief about quality, where the prior belief is not sufficient to support a sale, and the opposite case of a favorable prior. Here (and in the experiment) we focus on the more interesting case of an unfavorable prior where information transmission is necessary to realize the efficiency gains from trade. The introduction of noise greatly reduces the number of equilibria. There is always a pooling equilibrium where both types of sellers refrain from signaling whereas pooling on positive levels of signal costs cannot occur. Remarkably, for low levels of noise a pure strategy separating equilibrium ceases to exist. In a separating equilibrium, the buyer buys if and only if she receives a signal higher than a cutoff. With a low level of noise, this cutoff is much lower than the one used in the case without noise. It therefore becomes attractive for the low-quality seller to jump from providing zero signal costs to his interior optimum. This undermines the logic of the separating equilibrium in which the low-quality seller should refrain from signaling. The separating equilibrium only reappears for intermediate levels of noise, where the signal costs chosen by the high-quality type increase with the noise up to a ceiling. High noise levels force (high-quality) sellers to choose high signal costs, just as in the examples mentioned above. For very high levels of noise, the separating equilibrium disappears forever.

In our experimental design we vary the level of noise between our four treatments: from no noise to low noise (without a separating equilibrium) to intermediate noise (with a unique separating equilibrium based on an intermediate level of signal costs) to high noise (with a unique separating equilibrium based on a high level of signal costs). Our design allows us to address three questions: (i) Do subjects indeed refrain from separating for a low level of noise that prevents separation in theory?, (ii) Do the signal costs chosen by the (high-quality) seller increase with noise and, if so, do these costs increase monotonically or only for intermediate and high noise as predicted by theory?, and (iii) Does noise work as an empirical selection device? Regarding the last question, our conjecture was that noise works in favor of pooling, because separation becomes relatively less attractive when the noise in the signal increases.

We obtain the following experimental results. For no, low, and intermediate noise, subjects tend to separate. For high levels of noise some matching groups still 
play according to the logic of a separating equilibrium, but the majority switches to pooling on no signal costs. Conditional on aiming for separation, high-quality sellers' signal costs increase monotonically with the noise in the signal. Very high signal costs are occasionally observed when the noise in the signal is large.

To the extent that our results are not predicted by theory, we propose a sensible behavioral explanation. In particular, the intuitive anomaly that subjects separate in the low noise treatment where according to theory separation cannot be supported in equilibrium, can be understood as follows. With only a low amount of noise buyers initially behave as if there is no noise and thus use a higher cutoff than prescribed by equilibrium play. Sellers best respond and choose separating signal costs. Buyers feel no pressure to change their disequilibrium behavior, because the noise in the signal smoothes their expected payoffs and best responding does not noticeably increase their payoffs. Therefore, separation does not unravel.

Although a large literature on signaling games exists, noisy signaling has received little attention up till now. The path-breaking paper here is by Matthews and Mirman (1983), who include noisy signals in a (signaling) model of limit pricing. Within this context they show that the introduction of noise has substantial implications for the equilibrium predictions. Another important contribution is by Carlsson and Dasgupta (1997), who propose using vanishing noise as an equilibrium selection device in signaling games without noise. We consider the effect of noise in Spence's original pure signaling model in which signaling constitutes a pure social waste. In this noisy signaling game separating and pooling equilibria coexist, in contrast to the noisy signaling games of Matthews and Mirman and Carlsson and Dasgupta that only allow for separating equilibria. An important result is that only in the setup of Spence the existence of a separating equilibrium depends on the amount of (non-vanishing) noise in the signal. We are therefore able to study an issue that these earlier papers did not address, viz. how the occurrence of different types of equilibria (i.e. pooling vs. separating) varies with the level of noise. In the next section we will elaborate further on the distinctive features of our theoretical analysis.

Previous experimental papers on signaling have also searched for empirical equilibrium selection devices (in a noise-free context). Miller and Plott (1985) investigated signaling in a rich market institution, where sellers chose prices as well as costly quality increments to the product. In markets where the signaling costs were relatively low, market outcomes tended to converge to the separating equilibrium. Usually, high-quality sellers started with inefficiently high signaling costs before they converged to the minimum level that distinguished them from 
the low-quality types. Brandts and Holt (1992) studied the predictive power of belief-based equilibrium refinements in a game that modelled workers' choices for education and employers' subsequent hiring decisions. In early sessions, they found that play converged to the intuitive pooling equilibrium. Having studied the dynamics in the sessions, they were able to alter the parameters such that play tended to converge to the unintuitive pooling equilibrium. Cooper, Garvin and Kagel (1997a, 1997b) investigated a limit pricing game where low-cost monopolists had incentives to deter entry by high-cost monopolists. Subjects started at their "myopic optima", which allowed entrants to infer the monopolist's actual type and to act accordingly. This encouraged high-type monopolists to pool with the low-cost types. If no pooling equilibrium existed, initial attempts at pooling were shattered and play converged to a separating equilibrium. None of these papers considered the possibility that noise in the signal might function as a selection device. ${ }^{3}$

The remainder of our paper is organized in the following way. Section 2 provides a detailed description of the game and the theoretical analysis. Section 3 describes the experimental design and procedures. Section 4 presents the experimental results and section 5 concludes.

\section{Theory}

\subsection{The noisy signaling game}

We consider a simple signaling game between an informed seller and an uninformed buyer. The seller can be of two types, either good or bad; $p \equiv \operatorname{Pr}($ good $)$, with $0<p<1$, denotes the buyer's prior belief that the seller is of the good type. The seller first chooses his message $m \in[0, \infty)$ at signal costs $m$. The buyer then observes a noisy signal $z \in \mathbb{R}$, i.e. we assume that she observes $m$ with some additive noise:

$$
z=m+\sigma \cdot \varepsilon .
$$

\footnotetext{
${ }^{3}$ There are, however, experimental papers that investigated noisy communication in other games. Güth, Müller and Spiegel (2006) investigate the effects of noisy leadership in a sequential duopoly game. Aoyagi and Fréchette (2006) study collusion in a repeated prisoners' dilemma game where the opponent's past actions are imperfectly revealed in a noisy public signal. Feltovich, Harbaugh and To (2002) consider a signaling game experiment in which the receiver (in contrast to our setup) does perfectly observe the message chosen by the sender, but also receives some exogenous noisy information.
} 
Here $\varepsilon$ is a random variable with distribution function $F: \mathbb{R} \rightarrow[0,1]$ and $\sigma \geq 0$ reflects a scaling parameter to account for changes in the amount of noise. In line with Matthews and Mirman (1983) and Carlsson and Dasgupta (1997), we assume that the density $f$ is continuous and strictly positive everywhere. Moreover, like them we also assume that the conditional density of $z$ given $m$ satisfies the strict monotone likelihood ratio property (MLRP). ${ }^{4}$ Intuitively, this means that higher signal costs $m$ become more likely when the observed signal $z$ increases.

Having observed signal $z$, the buyer decides whether to buy or refrain from buying the product. The latter yields her 0 , irrespective of the seller's type. If the buyer decides to buy she obtains a payoff equal to $x>0$ when the seller is of the good type and $-y<0$ when he is of the bad type. The seller always bears the signal costs of his message choice $m$. Apart from that, the good (bad) type seller obtains a gross payoff of $g(b)$ from a sale. We assume that $g>b>0$, i.e. the sorting condition is satisfied. Table 1 summarizes these payoffs. Both seller and buyer are assumed to be risk-neutral.

\section{[ Insert Table 1 about here ]}

Note that our setup is isomorphic to the original one of Spence (1973) when we divide the seller's payoffs by his type $t \in\{b, g\}$; i.e. the seller obtains $1-\frac{m}{t}$ when the buyer buys and $-\frac{m}{t}$ if she does not. In this alternative specification the two seller types do not differ in their benefits of a sale, but rather in their (marginal) costs of producing message $m$ (which corresponds to the level of education in Spence's original formulation). Because this is just a normalization, equilibrium predictions are exactly the same.

\subsection{Equilibrium analysis}

From Spence (1973) it is well-known that without noise $(\sigma=0)$ there are many Perfect Bayesian equilibria. Among these are pooling equilibria in which both

\footnotetext{
${ }^{4}$ These assumptions facilitate the equilibrium analysis, because they imply that the buyer necessarily uses a cutoff strategy in any non-pooling equilibrium (cf. the Appendix). The important assumption here is that each possible signal comes from each type with strictly positive probability. As long as this assumption remains fulfilled, the analysis may be generalized to the case where signals have bounded support. In applications where negative signals are problematic, one may then choose to normalize the game such that only positive signals are possible. Alternatively, negative signals may be natural in applications where the costs are not exclusively monetary. As Spence (1973, p. 359) already noticed: "Signaling costs are to be interpreted broadly to include psychic and other costs..." Negative signals are also less problematic when one takes the seller's consumption good value of signaling into account: "The signal cost function does, in principle, capture education as a consumption good, an effect that simply reduces the cost of education" (cf. Spence, 1973, p. 364).
} 
seller types choose $m=0$ and separating equilibria in which the bad type chooses $m=0$ and the good type chooses some level of signal costs $m_{g} \in[b, g]$. All these equilibria exist independent of prior belief $p$. Moreover, when the buyer would buy in the absence of additional information - i.e., $p>\beta^{*} \equiv \frac{y}{x+y}-$ also pooling on any $m \in(0, b]$ can be supported as equilibrium.

Introducing noise by letting $\sigma>0$ narrows down the equilibrium set considerably. First, pooling on some $m>0$ cannot occur any longer. If both seller types choose the same signal costs $m$, then the buyer's posterior belief necessarily equals her prior for any signal $z$ observed. Her buying decision is then fully determined by her prior belief and independent of the signal received. Given this, the seller lacks any incentive to (stochastically) increase the signal and therefore only pooling on $m=0$ can occur. Second, adding noise also severely restricts the set of separating equilibria. We illustrate this by focusing on the case considered in the experiment where $F$ equals the standard normal distribution $N(0,1)$ (which will be denoted by $\Phi)$. In the Appendix we show that all the results discussed here generalize to any distribution function $F$ that satisfies the assumptions made in the previous subsection. Proofs of propositions are relegated to this Appendix as well.

We first consider pure strategy equilibria before we deal with mixed strategy equilibria. Proposition 1 below characterizes the set of separating equilibria that may exist besides pooling on $m=0$.

Proposition 1. Let $F=\Phi$ and assume that players are restricted to use pure strategies. (i) A pooling equilibrium in which both seller types choose $m=0$ always exists. In this equilibrium the buyer never [always] buys when $p<[>$ ]$\beta^{*} \equiv \frac{y}{x+y}$. Pooling on some $m>0$ cannot occur. (ii) Generically, i.e. for all $p \neq \frac{\beta^{*} \cdot g}{\left(1-\beta^{*}\right) \cdot b+\beta^{*} \cdot g}$, it holds that in any separating equilibrium the bad type of seller chooses $m=0$ whereas the good type chooses some positive level of signal costs $m=m_{g}>0$. The buyer buys if $z>z^{*}$ and refrains from buying otherwise. Signal costs $m_{g}$ and cutoff signal $z^{*}$ are given by:

$$
\begin{gathered}
m_{g}=z^{*}+\sqrt{2 \sigma^{2} \cdot\left[\ln \left(\frac{g}{\sigma \sqrt{2 \pi}}\right)\right]} \\
z^{*}=z_{h}^{*} \equiv \sqrt{2 \sigma^{2} \cdot\left[\ln \left(\frac{g}{\sigma \sqrt{2 \pi}}\right)-\ln \left(\frac{p\left(1-\beta^{*}\right)}{(1-p) \beta^{*}}\right)\right]} \quad \text { if } p \leq \beta^{*}
\end{gathered}
$$




$$
z^{*}=z_{h}^{*} \text { or } z^{*}=z_{l}^{*} \equiv-z_{h}^{*} \quad \text { if } p>\beta^{*}
$$

Note that Proposition 1 only characterizes the set of pure strategy equilibria. Conditions under which the separating equilibria indeed do exist will be discussed shortly.

The intuition behind the separating equilibria of Proposition 1 is as follows. Given that the noise distribution satisfies MLRP, the buyer necessarily uses a cutoff strategy; the seller's product is bought if and only if a signal larger than some cutoff level $z^{*}$ is observed. For a given value of $z^{*}$, the equilibrium level of signal costs $m_{g}$ the good type seller chooses then follows from equalizing the marginal benefits of raising $m$ with the marginal costs (equal to one) of doing so. This yields expression (1). ${ }^{5}$ The exact value of cutoff $z^{*}$ subsequently follows from the requirement that the buyer's posterior belief after observing $z^{*}$ should make her indifferent between buying or not. Because posterior beliefs are determined by Bayes' rule everywhere, this requirement puts some strong characterizing restrictions on the player's equilibrium strategies. Expressions (2) and (3) follow from these. As the latter expression makes clear, for $p>\beta^{*}$ there are two solutions for $z^{*}$, so actually two separating equilibria may potentially exist side by side.

Our next proposition concerns the actual existence of a separating equilibrium. In this regard we are particularly interested in how the amount of noise - as reflected by parameter $\sigma$ - affects existence.

Proposition 2. Let $F=\Phi$ and assume that players are restricted to use pure strategies. A necessary condition for a separating equilibrium to exist is that $\sigma \leq$ $\frac{g}{\sqrt{2 \pi}} \cdot \min \left\{\frac{(1-p) \beta^{*}}{p\left(1-\beta^{*}\right)}, 1\right\} \equiv \bar{\sigma}$. Assuming $\sigma \leq \bar{\sigma}$, it holds that:

(i) $p \leq \beta^{*}$ : a separating equilibrium does not exist if $\sigma$ becomes sufficiently small;

(ii) $\beta^{*}<p<\frac{\beta^{*} \cdot g}{\left(1-\beta^{*}\right) \cdot b+\beta^{*} \cdot g}$ : a separating equilibrium always exists. For this equilibrium it holds that $\lim _{\sigma \downarrow 0} m_{g}=0$;

(iii) $p>\frac{\beta^{*} \cdot g}{\left(1-\beta^{*}\right) \cdot b+\beta^{*} \cdot g}$ : a separating equilibrium does not exist.

Two main observations follow from Proposition 2. First, when there is a lot of noise separation cannot occur; for $\sigma>\bar{\sigma}$ separating simply becomes too difficult

\footnotetext{
${ }^{5}$ In fact, this expression incorporates the second order condition as well; from the SOC it follows that $m_{g}$ necessarily exceeds $z^{*}$.
} 
or too costly for the good type seller. Formally this can be understood from expressions (1) and (2). The terms within square brackets become negative for $\sigma$ sufficiently large and no sensible solutions for $m_{g}$ and $z^{*}$ exist.

Second, a separating equilibrium also fails to exist when only a small amount of noise is introduced in the unfavorable prior case $p \leq \beta^{*}$. A priori the buyer then refrains from buying, but observing a small positive signal would already induce her to change her mind. (Formally, cutoff $z_{h}^{*}$ as given by (2) is low when $\sigma$ is small.) But given that the buyer is persuaded so easily, the bad type seller may want to deviate from $m=0$. Similar to (1) above, his best candidate deviation level equals:

$$
m_{b}=z^{*}+\sqrt{2 \sigma^{2} \cdot\left[\ln \left(\frac{b}{\sigma \sqrt{2 \pi}}\right)\right]}
$$

One requirement for a separating equilibrium to exist is thus that $m=m_{b}$ should yield the bad type seller weakly less than $m=0$. This reduces to: ${ }^{6}$

$$
b \cdot\left(\Phi\left(\frac{z^{*}}{\sigma}\right)-\Phi\left(\frac{z^{*}-m_{b}}{\sigma}\right)\right) \leq m_{b}
$$

Because this condition depends on $\Phi$, no closed form expression for the cutoff value on $\sigma$ can be obtained. But it can be shown that it is necessarily violated for $\sigma$ small enough. $^{7}$

The prediction that just a small amount of noise destroys separation is a priori somewhat counter-intuitive. To better understand the underlying driving force, let the payoff parameters be such as in the experiment (where $p=0.5<0.6=\beta^{*}$, see Table 2) and consider first the least cost separating equilibrium of the no noise game. In this so-called Riley outcome the bad type seller chooses $m=0$ and the good type $m=90(=b)$. The buyer buys only if a signal of $z=90$ or higher is observed. Figure 1a reflects both the densities of the signals generated by the two

\footnotetext{
${ }^{6}$ The mirror image requirement for the good type seller is that he should not have an incentive to deviate from $m_{g}$ towards $m=0$, i.e. $g \cdot\left(\Phi\left(\frac{z^{*}}{\sigma}\right)-\Phi\left(\frac{z^{*}-m_{g}}{\sigma}\right)\right) \geq m_{g}$ is needed. This actually has a bite in case (i) of Proposition 2. There a separating equilibrium may not exist when $\sigma$ becomes large within the relevant interval $(0, \bar{\sigma}]$.

${ }^{7}$ Also in part (ii) where $\beta^{*}<p<\frac{\beta^{*} \cdot g}{\left(1-\beta^{*}\right) \cdot b+\beta^{*} \cdot g}$ the separating equilibrium based on $z_{h}^{*}$ vanishes for $\sigma$ sufficiently small. In that case, however, an equilibrium based on $z_{l}^{*}<0$ always exists (given that $\sigma \leq \bar{\sigma}$ ). In this equilibrium the buyer a priori intends to buy and even receiving a moderately negative signal does not lead her to behave differently. The bad type seller therefore does not have an incentive to deviate from $m=0$. Part (iii) of Proposition 2 can be intuitively understood from considering how cutoff level $z^{*}$ (either equal to $z_{l}^{*}$ or $z_{h}^{*}$ ) varies with prior belief $p$. Because $z^{*}$ is close to zero when $p$ is high, the bad type seller obtains a strong incentive to deviate from $m=0$, i.e. no-deviation condition (5) is violated. This upsets the separating equilibria.
} 
seller types (labelled $f_{B}$ and $f_{G}$ ) and the buyer's posterior belief $\beta(z)$. The signal densities are degenerate at $z=0$ and $z=90$, respectively. This implies that $\beta(z)$ is determined by Bayes' rule only for these two values of $z$. To support the equilibrium, out-of-equilibrium beliefs must be such that $\beta(z) \leq \beta^{*}$ for $z<90$. The figure depicts the equilibrium where $\beta(z)=0$ in that case. Importantly, for any signal between 0 and 90 the buyer may hold skeptical beliefs that the signal quite likely came from the bad type seller, even when it is close to 90 .

\section{[ Insert Figure 1 about here ]}

Now consider what happens if some noise is added, of size $\sigma=10$ say. Naively one would then expect that the good type seller simply moves up his signal cost a bit, to 100 say, while the bad type stays put. Figure 1b depicts this situation. In contrast to the no noise case, the two signal densities are now non-degenerate and posterior beliefs $\beta(z)$ for in between signals $0<z<100$ now follow from Bayes' rule. Given the highly concentrated signal densities, signals closest to 0 most likely come from the bad type while signals closest to 100 most likely come from the good type. The actual cutoff for which $\beta\left(z^{*}\right)=\beta^{*}$ is roughly in the middle at $z^{*} \approx 50.4$. But if the buyer uses this cutoff value, the bad type seller wants to jump away from $m=0$ towards $m_{b} \approx 66.4$ (cf. expression (4)). This destroys the separation outcome. Another destabilizing factor is that for $z^{*} \approx 50.4$ the good type seller also wants to deviate from 100 to $m_{g} \approx 73.9$.

From the above it follows that crucial for separation to unravel when some noise is introduced, is that the buyer realizes that she should set a (much) lower cutoff level above which she decides to buy. If she does not do so and keeps the cutoff at $z^{*}=90$ (or somewhat higher), the two seller types still have an incentive to separate.

\section{[ Insert Table 2 about here ]}

The overall comparative statics in $\sigma$ are illustrated in Table 2. For $\sigma$ roughly below 24 separation cannot occur. The same holds for high amounts of noise (viz. $\sigma$ roughly above 142) whereas for in between levels a separating equilibrium exists besides the pooling one. ${ }^{8}$ In these separating equilibria the level of signal costs chosen by the good type increases with noise (for $\sigma$ not too high). This level $m_{g}$

\footnotetext{
${ }^{8}$ Note that standard belief-based equilibrium refinements (like the intuitive criterion of Cho and Kreps, 1987) are ineffective in our setup, because for $\sigma>0$ there are no out-of-equilibrium beliefs.
} 
can actually become quite large relative to $g$ and $b$, as the case with $\sigma=120$ illustrates. The good type thus may be 'forced' to use very costly signals.

Mixed strategy equilibria Up till now we have assumed that seller and buyer may only use pure strategies. Theorem 3 in the Appendix shows that if we allow them to use mixed strategies as well, two additional types of equilibria may potentially exist as well. In the first type of mixed equilibrium the bad type seller mixes between $m=0$ and signal costs $m_{b}$ as given in (4), whereas the good type chooses $m_{g}$ from expression (1) for sure. The bad type's mixing probability $q_{b} \equiv \operatorname{Pr}\left(m=m_{b}\right)$ follows from the requirement that observing $z^{*}$ should make the buyer indifferent (i.e. $\beta\left(z^{*}\right)=\beta^{*}$ ). For the bad type to be willing to mix, condition (5) now has to hold with equality. Given the appearance of $\Phi$ here, the resulting equilibrium values of $z^{*}, m_{b}$ and $m_{g}$ have to be calculated numerically. Table 3 provides an overview for some relevant parameter values. For the values of $\sigma$ considered in the experiment, this mixed equilibrium appears to exist for $\sigma=10$ only. ${ }^{9}$ It exists for smaller amounts of noise as well. In fact, it holds that $m_{g}$ and $m_{b}$ tend to $b$ as $\sigma$ tends to 0 and that $\lim _{\sigma \downarrow 0} q_{b}>0$. When the noise vanishes this equilibrium thus converges to a mixed equilibrium of the no noise game that is insufficiently revealing; with strictly positive probability the buyer takes the opposite decision of what she would do under complete information. ${ }^{10}$

In the other mixed strategy equilibrium the good type seller mixes between $m=0$ and $m=m_{g}$, while the bad type chooses $m=0$ for sure. For the good type to be indifferent it now must hold that $g \cdot\left(\Phi\left(\frac{z^{*}}{\sigma}\right)-\Phi\left(\frac{z^{*}-m_{g}}{\sigma}\right)\right)=m_{g}$, so also here closed form expressions cannot be obtained. What can be shown theoretically, however, is that this equilibrium converges to a pooling (on $m=0$ ) equilibrium when the noise becomes small, i.e. $\lim _{\sigma \downarrow 0} q_{g}=0$. Moreover, signal costs $m_{g}$ become large for low values of $\sigma: \lim _{\sigma \downarrow 0} m_{g}=\lim _{\sigma \downarrow 0} z^{*}=g$. The parametric examples in Table 3 illustrate this.

\section{[ Insert Table 3 about here ]}

The first mixed strategy equilibrium discussed above challenges the prediction that for low values of $\sigma$ separation will not occur. In the other mixed equilibrium the positive signal costs $m_{g}$ decrease with $\sigma$. Because these predictions run counter to the main predictions based on pure strategy equilibria only, we explicitly check

\footnotetext{
${ }^{9}$ When $\sigma$ becomes too large, the defining equation of $q_{b}$ results in a negative value.

${ }^{10}$ In this particular case the buyer always buys if $z=b$ is observed, although with positive (but small) probability the seller is of the bad type.
} 
in our empirical analysis whether actual behavior is consistent with these mixed strategy equilibria.

\subsection{Related theoretical literature}

Matthews and Mirman (1983) consider a limit pricing model with a potential entrant and an incumbent monopolist who is privately informed about industry conditions. The actual price the incumbent charges depends on both his output choice and a random demand shock that occurs after the output decision. Owing to this noise, equilibria are obtained that differ from standard signaling game equilibria in three ways: (i) there is a great reduction in the number of equilibria, (ii) (separating) equilibrium strategies now directly depend on prior beliefs, and (iii) different amounts of information are revealed in different separating equilibria, leading to richer comparative statics. ${ }^{11}$

These three features apply in our setting as well. First, with noise (generically) only five different equilibria exist at most, as opposed to the continuum of equilibria in the no noise case. For a range of parameter values (e.g. $p$ sufficiently high) the equilibrium is even unique. Second, as expressions (1) through (3) reveal, separating equilibrium strategies directly vary with prior belief $p$. For instance, cutoff value $z_{h}^{*}$ decreases with $p$, implying that the buyer is more easily persuaded to buy if she is already more inclined to do so a priori. This seems a much more intuitive prediction than the irrelevance of $p$ for the required level of separation that the no-noise case predicts. Similar remarks apply to variations in $g$. Third, with noise even a separating equilibrium is insufficiently revealing. Different separating equilibria may therefore lead to different amounts of information being revealed. In our setting two different separating equilibria may actually exist side by side when the prior is favorable. In the one based on $z_{h}^{*}$ the signal costs that the two seller types choose are more dispersed and the buyer obtains more information than in the one based on $z_{l}^{*}$ (cf. Proposition 1).

Carlsson and Dasgupta (1997) focus on equilibrium selection in signaling games

\footnotetext{
${ }^{11}$ The introduction of noise may have profound implications in complete information contexts as well. Bagwell (1995) studies a noisy leader game in which a follower observes the actual choice a leader made with some noise. The striking result he obtains is that the standard first-mover advantage then completely disappears. As pointed out by van Damme and Hurkens (1997), this conclusion depends on the restriction to pure strategies. When the noise is small, there always exists a mixed strategy equilibrium that approximates the standard Stackelberg outcome of the game without noise. Also the assumption of complete information is crucial. Maggi (1999) shows that the value of commitment reappears when the leader's choice is based on private information that is payoff-irrelevant for the follower. The latter assumption allows Maggi to abstract away from signaling considerations, which are the main focus of this paper.
} 
without noise, by studying the limiting set of 'noise-proof' equilibria that results from letting the noise vanish. Among other things, they show that every noiseproof equilibrium of the no noise game is necessarily insufficiently revealing. Our theoretical analysis replicates this finding for our setup. In particular, by letting $\sigma$ go to zero Theorems 2 and 3 in the Appendix show that there exist only two noise proof equilibria in the no noise game, viz. pooling on $m=0$ and the mixed equilibrium described before in which only the bad type mixes between $m=0$ and $m_{b}=b$. Both are insufficiently revealing, as with positive probability the buyer takes a decision that she will regret ex post. ${ }^{12}$

Despite these similarities, our theoretical findings differ in some other important respects from these two earlier studies. In both Matthews and Mirman (1983) and Carlsson and Dasgupta (1997) the equilibria of the noisy signaling games they study are always separating. ${ }^{13}$ In contrast, our setup allows for pooling equilibria as well. At the same time, only in our game the existence of a separating equilibrium is not guaranteed and depends on the amount of noise in the signal. Unlike these previous authors, therefore, we are able to study the question of how the existence of different types of equilibria varies with noise.

The main driving force why we obtain results that differ from both Matthews and Mirman (1983) and Carlsson and Dasgupta (1997) is that in these earlier papers the seller's (message) choice actually serves two purposes. Besides a pure signaling function geared towards influencing the behavior of the receiver, the seller's choice also allows him to optimally adapt to changing circumstances. In Matthews and Mirman this adaptation purpose for example follows from the fact that the output choice (message) of the incumbent monopolist (seller) varies with industry conditions (type), even when the receiver (potential entrant) is fully in-

\footnotetext{
${ }^{12}$ Some other main findings of Carlsson and Dasgupta (1997) do not carry over to our setting. For example, by Proposition 1(a) it follows that pooling on $m=0$ is noise-proof. But for $p<\beta^{*}$ this equilibrium does not survive the never-a-weak-best response (NWBR) refinement in the game without noise. Unlike in Carlsson and Dasgupta (1997), therefore, in our setup not every noise-proof equilibrium satisfies NWBR. Likewise, we do not find a unique noise proof equilibrium whereas in Carlsson and Dasgupta it "often" is.

${ }^{13}$ The same holds for the noisy signaling model used by Kanodia, Singh and Spero (2005) to study a firm's optimal investment in the presence of capital market imperfections. Calveras (2003) embeds a noisy signaling game in a model of a bank that can manipulate the noisy information a regulator observes. His signaling subgame allows for both pooling and separating equilibria (see his Proposition 3). Apart from his model being much more specific than ours, Calveras does not study how the existence of the different types of equilibria varies with the amount of noise nor does he consider mixed strategy equilibria. The latter also applies to Hetzendorf's (1993) analysis of noisy advertising in the multi-dimensional signaling model of Milgrom and Roberts (1986). The purpose of his study is to show that in the presence of noise, no separating equilibrium exists in which prices and advertising are simultaneously used as informative signals.
} 
formed on these industry conditions. Optimal output is higher the more favorable industry conditions are. The seller's output choice therefore does not perform a pure signaling function alone. The same applies for the setup in Carlsson and Dasgupta (1997). ${ }^{14}$ The important consequence of this two-folded purpose is that (in the noisy games) the seller's best response correspondence is strictly monotonic in his type (cf. Proposition 3.1 in Carlsson and Dasgupta, 1997). Loosely put, the two purposes together pull sellers towards separation.

In our setup the seller's message choice only serves a pure signaling function, such that (costly) signaling constitutes a pure social waste. ${ }^{15}$ If in our game the seller's type would be public information, the seller's ('message') choice would be independent of his type and equal $m=0$ for both types. ${ }^{16}$ As a result, sellers' best responses are only weakly monotonic in types (cf. the proof of Lemma 1 in the Appendix). The pull towards separation is therefore much weaker. This implies in turn that our setup allows for pooling equilibria as well while at the same time the existence of a separating equilibrium is not guaranteed.

\section{$3 \quad$ Experimental design and procedures}

The computerized experiment was run at the University of Amsterdam where subjects were recruited from the student population. Subjects read the on-screen instructions at their own pace. At the end of the instructions, subjects had to answer some test questions correctly before they could proceed. They also received a summary of the instructions on paper. ${ }^{17}$ Subjects knew that the experiment consisted of two parts. Part 1 lasted for 40 periods. In part 2 subjects formulated a

\footnotetext{
${ }^{14}$ Let $\pi$ denote the probability with which the buyer buys and $u(t, m, \pi)$ the sender's utility. Carlsson and Dasgupta (1997) assume that $\frac{\partial u(t, m, \pi)}{\partial m}=0$ has a unique (interior) solution $m_{\pi}(t)$, with $m_{\pi}(t)$ strictly increasing in $t$ (cf. Assumptions (U3) through (U5) on p. 443). Note that in our setup we have $u(t, m, \pi)=t \cdot \pi-m$, so $\frac{\partial u(t, m, \pi)}{\partial m}=-1$ and this assumption is not met.

${ }^{15}$ By focusing on costly signaling our setup differs from "cheap talk" games in which messages are costless. See Blume, Board and Kawamura (2007) for an analysis of the impact of introducing noise on the amount of information transmission in the cheap talk model of Crawford and Sobel (1982). Landeras and Pérez de Villarreal (2005) introduce noise into a screening model in which the uninformed party moves first.

${ }^{16}$ Our different results are thus not due to the fact that in our setup the marginal costs of raising $m$ are independent of the seller's type, as one a priori might have expected. As explained in the main text, our setup is completely isomorphic to the case where seller's utility equals $u(t, m, \pi)=\pi-\frac{m}{t}$, with $\pi$ the probability with which the buyer buys. Just as in Carlsson and Dasgupta (1997), in this specification marginal costs are type dependent.

${ }^{17}$ The instructions are available at the following url: http://www1.fee.uva.nl/creed/pdffiles/INSTRUCTIONsignal.pdf. The instructions file also contains the figures that were used to explain the normal distribution of the noise term to the subjects (see below).
} 
strategy that automatically determined their play in another 10 periods. Subjects received instructions for the second part only after the first part was finished. At the beginning of the experiment, subjects received a starting capital of 5000 points. Their period earnings (losses) were added to (subtracted from) this starting capital. At the end of the experiment, points were exchanged into euros at a rate of 1 euro for 250 points. In 1.5 to 2 hours, a total of 184 subjects earned on average 37.05 euros with a standard error of 10.89 .

At the start of the first part subjects were assigned to the role of seller or the role of buyer. Throughout the whole experiment subjects kept the same role. Each period, sellers and buyers were randomly matched in pairs within a (fixed) matching group of 8 subjects. Subjects knew that they were never matched with the same subject twice in a row. In most sessions, we ran 2 matching groups simultaneously. At the start of a period, the seller was privately informed of the quality of his product. In each matching group, 4 products had high quality and 4 products had low quality. Thus, the prior probability of a high-quality product was 0.5 , a fact that was communicated to all subjects. The quality of the product of a seller in a given period was independent of the quality of his product in another period. After observing the quality of his product, the seller chose a signal cost, an integer amount between 0 and 400. The computer added a noise term, an independent draw from a $N\left(0, \sigma^{2}\right)$ distribution to the signal cost, and communicated the resulting signal, but not the signal cost nor the noise term, to the buyer. We communicated the density of the normal distribution with the help of a figure and some explanatory remarks about symmetry and confidence intervals. The buyer decided whether or not to buy the product, after which the payoffs of the pair were determined. The payoff table was common information to the subjects.

At the end of a period, both players were informed of the quality, the signal cost and the signal. In addition, subjects could view a social history window at the bottom of the screen that showed the results of all pairs in their own matching group for the last 10 periods. For buyers, the screen was ordered on signal (from high to low), quality (from high to low), signal cost (from high to low) and buy-decision (from yes to no), respectively. Subjects could recognize their own previous results as these were printed against a different, light-gray background. Figure 2a shows a snapshot of this window. Figure $2 \mathrm{~b}$ shows the social history window for sellers, which was ordered on signal cost (from high to low), signal (from high to low), buy-decision (from yes to no) and quality (from high to low), respectively. We provided this information because it allows 
subjects to learn faster. Our paper deals with the topic of equilibrium selection and the comparative statics of the equilibrium predictions with respect to the amount of noise. To address these issues play must converge to equilibrium in the first place. We think that, compared to the world outside of the laboratory, the superior information provided to subjects balances their lack of experience with the game. A similar social history (on black board) was first provided in a signaling experiment of Miller and Plott (1985), who introduced it in the later sessions to help subjects recognize the relationship between types and choices. ${ }^{18}$

The variance of the error term in the signal $\left(\sigma^{2}\right)$ was the only variable that varied between the 4 treatments. We refer to the treatments as $\sigma_{0}, \sigma_{10}, \sigma_{40}$ and $\sigma_{120}$. Table 4 summarizes the details of the experimental design. Each subject participated in one treatment only. We correctly anticipated that behavior would become more volatile for higher noise levels in the signals. Therefore, we decided to collect a larger number of observations for the treatments with the higher noise levels.

\section{[ Insert Figure 2 about here ] [ Insert Table 4 about here ]}

In the second part of the experiment, we asked subjects to formulate a strategy for periods 41-50. Buyers were asked to provide a cutoff level for the signal received, at and above (below) which they would (not) buy the product. They could also indicate that they would never or always buy the product, independent of the signal. Sellers were asked to choose a signal cost for high-quality products as well as for low-quality products. We explicitly mentioned that it was up to the seller to decide whether he wanted to choose the same signal costs for high and low quality or different amounts. We emphasized that otherwise the game was exactly the same as the one they played in the first 40 periods. When all subjects had chosen their strategies, the computer automatically played out the final 10 periods.

\section{Experimental results}

We present the experimental results in two parts. First, we deal with the results at the aggregate level. We delve into the question how noise affects the equilibria

\footnotetext{
${ }^{18}$ Other papers have used role reversion to accomplish this. After senders have become receivers, it becomes easier for them to interpret the meaning of a signal (e.g., Brandts and Holt, 1992).
} 
selected and the level of signal costs chosen. In the second part, we zoom in on the behavior of our subjects and provide a coherent explanation of the main results.

\subsection{Equilibrium selection and increasing noise}

We anticipated that it would take a while before subjects would converge to equilibrium, because of confusion and out-of equilibrium play in the early periods. For instance, confusion may cause low-quality sellers to choose dominated signal costs above 90 . In periods $1-10,4.3 \%$ of the low type sellers do so versus $1.5 \%$ after period 20. This suggests that even in the early periods confusion played only a minor role. Nevertheless, we decided to stick to our original plan and focus on the data of the second half of part 1 (periods 21-40). It turns out that the results for the strategy method confirm the results of part 1 to a large extent. We have chosen to limit the report of the results on the strategy method to the extent that they provide additional insight.

First we take a look at how sellers behaved. Figures 3a-3h present histograms of the signal costs chosen by high-quality sellers and low-quality sellers. In treatment $\sigma_{0}$, high-quality sellers most often chose a signal cost of 100 , followed by 91 . A large majority of $93.2 \%$ of submitted signal costs lied between 90 and 100. A total of $88.8 \%$ of the signal-costs submitted by low-quantity sellers in $\sigma_{0}$ equaled exactly 0 . In this treatment, sellers' behavior provides clean evidence for separation.

\section{[ Insert Figures 3a-3h about here ]}

Also in treatment $\sigma_{10}$ low-quality sellers overwhelmingly chose 0 , while highquality sellers chose to separate. The latter tended to send higher signal costs, also at a higher variance than in $\sigma_{0}: 86.5 \%$ of the submitted signal costs were in between 90 and 130. This pattern of higher and more volatile signal costs extends to treatment $\sigma_{40}$, where high-quality sellers separated with $79.6 \%$ of the signal costs lying between 100 and 160, while low-quality sellers stuck to 0 . The picture looks very different for treatment $\sigma_{120}$, though. With $40.9 \%$ of the high-quality sellers choosing a signal cost of exactly 0 , the focus of sellers' attention seemed to be on pooling. Still a considerable fraction of $41.6 \%$ of the signal costs was at 90 or above. High signal costs were very spread out. In contrast, low-quality sellers by and large chose a signal cost of 0 , like in the other treatments.

Very high signal costs were observed occasionally. In treatments $\sigma_{40}$ and $\sigma_{120}$, high-quality sellers chose signal costs of at least 230 in $4.2 \%$ and $5.6 \%$ of 
the cases, respectively. In the other treatments such high signal costs were never observed.

A key-prediction of the theoretical analysis is that conditional on the existence of a separating equilibrium, the signal costs should increase with noise as long as subjects coordinate on separating. Table 5 splits the submitted signal costs into pooling (below 90) and separating (at and above 90), for low-quality sellers as well as high-quality sellers. ${ }^{19}$ Conditional on a separating signal cost being sent, the signal cost indeed increased monotonically from on average 97.9 in $\sigma_{0}$ to 158.3 in $\sigma_{120}$. Using Mann-Whitney rank tests we find that the differences in signal costs between the treatments are all significant or weakly significant, except for the comparison between $\sigma_{40}$ and $\sigma_{120}$.

\section{[ Insert Table 5 about here ]}

The table foreshadows the main result regarding equilibrium selection. The relative frequency of pooling signals increases with noise. In treatments $\sigma_{0}, \sigma_{10}$, $\sigma_{40}$ and $\sigma_{120}$, high-quality senders submitted pooling signal costs in $0.6 \%, 2.0 \%$, $7.5 \%$ and $58.4 \%$ of the cases, respectively. In all treatments the overwhelming majority of low-quality sellers submitted signal costs of 0 . Thus, sellers aimed for separation in treatments $\sigma_{0}, \sigma_{10}$ and $\sigma_{40}$, while the results are mixed for treatment $\sigma_{120}$, where the pooling equilibrium attracted sellers more than the separating equilibrium did.

Table 5 also reveals two differences between equilibrium predictions and submitted signal costs. The first difference is that the submitted signal cost increased on average monotonically with noise, while theoretically a separating equilibrium does not exist in treatment $\sigma_{10}$. The second difference is that high-quality sellers' signal costs did not increase as rapidly with noise (from $\sigma_{40}$ to $\sigma_{120}$ ) as equilibrium predicts.

Of course, it only makes sense for sellers to play equilibrium when buyers play equilibrium. Therefore, a more relevant question is to what extent sellers chose best responses to the actual behavior of the buyers. We describe the latter by means of an empirical cutoff value $\widehat{z}^{*}$. In particular, we determined in each matching-group and each period which cutoff level $\widehat{z}^{*}$ for the buyers minimized the sum of the buyers' errors against that cutoff level in the 10 most recently

\footnotetext{
${ }^{19}$ This classification follows from the observation that in a separating equilibrium (if it exists), the good type seller always chooses a signal cost that exceeds 90 (see the case $p<\beta^{*}$ in Table 2). Positive signal costs below 90 thus cannot be interpreted as an attempt to separate and are therefore labelled pooling.
} 
completed periods of that particular group. ${ }^{20}$ We subsequently set the seller's empirical best response equal to the signal cost that maximized expected payoff given this cutoff level $\widehat{z}^{*}$ of the buyers. ${ }^{21}$ Often, there was a range of cutoff levels that fitted the data equally well. In those cases, we determined the best response to the maximum cutoff level in the optimal range and the best response to the average cutoff level in the optimal range. It turns out that the best response on the basis of the maximum cutoff level was closer to the actual signal cost than the one based on the average. Therefore, we report statistics based on the maximum.

Table 5 includes a column that reports the sellers' best responses. In agreement with the actual data, the best responses of high-quality sellers increased monotonically with noise, with lesser increments than the equilibrium-predictions. Note that the high-quality sellers' best response in treatment $\sigma_{10}$ equaled 117.9 , quite close to the actual data, despite the fact that a separating equilibrium does not exist here. We will come back to this remarkable finding in Subsection 4.2.

To assess whether subjects coordinated on a pooling or separating equilibrium, buyers and sellers' behavior have to be scrutinized simultaneously. First, we deal with the possibility that subjects played in accordance with the logic of a mixed equilibrium. As explained in Section 2, there are two types of mixed equilibria. The one where the good type mixes has two features that are incompatible with the data. The first one is that the comparative statics prediction is violated. According to this equilibrium, the positive signal cost chosen by the high-quality seller should decrease with (increasing) noise, while it actually increased. Second, in $\sigma_{120}$, the good type should mix between 0 with probability 0.75 and 290.95 with probability 0.25 . High-quality sellers submitted signal costs higher than 250 in only $2.2 \%$ of the cases, however. In all other treatments, the mixed equilibrium is observationally indistinguishable from pure pooling equilibria and we will deal with those later.

The next type of mixed equilibrium is the one where the low-quality seller mixes. This equilibrium exists in $\sigma_{10}$, but not in $\sigma_{40}$ nor in $\sigma_{120}$ (see Table 3). In $\sigma_{10}$, in 6 out of 200 cases low-quality sellers chose a signal cost larger than 0 , three times 70 and three times 80. According to the mixed equilibrium, bad type sellers should choose a signal cost 85 with probability 0.15 and 0 otherwise. So the positive signal costs are below the theoretically expected level. Moreover, the proportion of positive signal costs $(3 \%)$ falls considerably short of the theoretically

\footnotetext{
${ }^{20}$ Remember, sellers had access to a social history screen of 10 periods deep of their own matching-group, which also listed the behavior of the buyers.

${ }^{21}$ Depending on the level of $\widehat{z}^{*}$, the best response of the good [bad] type seller equals either signal costs of $m_{g}\left[m_{b}\right]$ as given by expression (1) [(4)], or zero signal costs.
} 
expected level (15\%). In fact, a binomial test rejects the hypothesis that the proportion of positive observations is in line with the theoretical prediction ( $p=$ 0.00). Choosing positive signal costs was not a great idea for bad type sellers: 5 out of these 6 positive signal costs led to signals above the equilibrium cutoff level of buyers, but only 3 actually led to a sale. Thus, sending these positive signal costs led to an average loss of 30 . These 6 observations are probably best interpreted as unsuccessful attempts of low-quality sellers to fool the buyers, or simply mistakes, instead of mixed-equilibrium play. We conclude that mixed equilibria do not organize the data well. In the remainder, we will focus on the pure strategy equilibria.

For each matching group, we computed the number of outcomes consistent with the pooling equilibrium and the number of outcomes consistent with the separating equilibrium. An outcome is consistent with the pooling equilibrium if and only if there was no sale. An outcome is consistent with the separating equilibrium if and only if the buyer's decision whether or not to buy was in accordance with the separating prediction that depended on the quality of the seller and the actual noise term in the signal. ${ }^{22}$ (Thus, an outcome may be consistent with both types of equilibria and also with neither type.) Table 6 lists for each matching group the extent to which actual play agreed with either of the equilibria in periods 21-40. In treatments $\sigma_{0}, \sigma_{10}$ and $\sigma_{40}$, the outcomes of all groups agreed much better with the separating equilibrium than with the pooling equilibrium. In treatment $\sigma_{120}$, the results were less clear-cut; either equilibrium attracted half of the groups. The results based on the strategy method in periods 41-50 were the same as the ones reported for periods 21-40, except that two of the groups (\#4 and \#5 in treatment $\left.\sigma_{120}\right)$ that were playing in accordance with separating in periods 21-40 switched to pooling in periods 41-50. In fact, in periods 21-40, for these two groups the separating equilibrium only predicted marginally more outcomes than the pooling equilibrium. Our interpretation is that these groups had not yet converged to equilibrium in periods 21-40. After unsuccessfully trying to establish separating play, subjects in these groups switched to pooling in the final 10 periods. Thus, in $\sigma_{120}$, at the end of the experiment 6 of the 8 matching-groups agreed with pooling and the 2 others with separating.

\section{[ Insert Table 6 about here ]}

\footnotetext{
${ }^{22}$ For $\sigma=10$ we defined an outcome consistent with separation if either (i) the seller is of the bad type and there is no sale, or (ii) the seller is of the good type and trade takes place. Here the definition of a separating outcome thus corresponds with the $\sigma=0$ case.
} 
Table 6 also shows how and to what extent actual results deviate from best responses. The procedure to calculate best responses for sellers was already explained above. For buyers we used the following procedure. In each matchinggroup and each period, we determined the average signal cost chosen by the good type sellers $\left(\widehat{m_{g}}\right)$ and the average signal cost chosen by the bad type sellers $\left(\widehat{m}_{b}\right)$ in the last 10 periods. Then we computed the best response cutoff level $z^{B R}$ given these average signal costs. ${ }^{23}$ This cutoff level and the received signal together determined the buyer's best response decision to buy or not. It is noteworthy that in most groups of the treatments with noisy signals high-quality sellers chose lower signal costs than the best response prediction. We will come back to this result in Subsection 4.2. Note also that buyers bought less than the best response model predicted in treatments $\sigma_{0}, \sigma_{10}$ and $\sigma_{40}$, but more than the best response model in treatment $\sigma_{120}$. This limits the scope for an explanatory role of risk-aversion. Risk averse buyers should use higher cutoff levels than risk neutral buyers and therefore buy less often. The data in $\sigma_{0}, \sigma_{10}$ and $\sigma_{40}$ deviate in the direction expected by risk aversion. In $\sigma_{120}$, the data deviate in the opposite direction, however.

We now take a closer look at buyers' behavior. For each individual buyer, we estimated her personal cutoff signal $\widehat{z}^{*}$ below which she did not buy. The cutoff level was set such that the number of errors against the cutoff level was minimized. Table 7 presents the data separately for the groups that were classified as pooling and the ones that were classified as separating in Table 6 . In $\sigma_{120}$, subjects in pooling groups employed much larger cutoff levels than subjects in separating groups. For the separating groups, subjects used higher cutoff levels than predicted by best response and equilibrium in treatments $\sigma_{0}, \sigma_{10}$ and $\sigma_{40}$, but lower cutoff levels in treatment $\sigma_{120}$. Overall, subjects' cutoff levels were not sufficiently responsive to the noise in the signal.

\section{[ Insert Table 7 about here ]}

\subsection{Explaining the results}

In the previous section we compared actual behavior to equilibrium behavior and best response. Playing best response is cognitively very demanding and it is very unlikely that boundedly rational subjects with limited time actually derived the best response functions for their role. In this section, we provide a behavioral

${ }^{23}$ This best response is given by $z^{B R}=\frac{1}{2}\left\{\left(\widehat{m_{g}}+\widehat{m_{b}}\right)+\frac{2 \sigma^{2} \ln \left[\frac{\beta^{*}(1-p)}{\left(1-\beta^{*}\right)}\right]}{\widehat{m_{g}-\widehat{m}_{b}}}\right\}$. 
explanation that requires less cognitive effort and contrast this explanation with actual behavior and best responses.

For buyers, we collected the 5 signals in the social history screen that were closest to the received signal in the current period and we counted how often those 5 signals were accompanied by high quality (remember, for buyers the history screen was ordered on the basis of signal, see Figure 2a). We took this statistic as a measure of the buyer's belief of the probability of high quality given the signal and set the behavioral response equal to the best response based on this belief. Actually, if 3 out of 5 signals were connected to high quality, the posterior probability of high quality was 0.6 and the buyer should be indifferent between buying and not buying. In those cases we looked at the relative frequency of high quality in the 3 closest signals and let this statistic decide the decision to buy or not buy (i.e., buy if and only if at least 2 out of 3 were high quality).

Table 8 presents buyers' decisions together with the best responses and the behavioral responses. It is striking how similar best and behavioral responses are. Aggregated across treatments, $89.3 \%$ of all buyers' decisions agree with best responses. The behavioral response explanation only performs marginally better, since it gets $90.2 \%$ of the actual decisions right. In $93.0 \%$ of the cases, the best response coincides with the behavioral response.

\section{[ Insert Table 8 about here ]}

Although behavioral and best responses trace buyers' behavior very well, one feature of the data has not yet been explained. Table 7 revealed that buyers' cutoff levels were not sufficiently responsive to the noise in the signal. Here follows a potential explanation. It is quite natural for buyers to start with a cutoff level of 90, the amount that the low-quality seller earns when his good is bought. The question is why in the treatments with noise buyers did not learn to change the cutoff sufficiently into the direction of the true best response. Figures $4 \mathrm{a}-4 \mathrm{~d}$ provide an answer to this question. For each treatment, these figures show the buyer's expected payoff and its volatility conditional on each possible cutoff level $z$, given the actual average signal costs chosen by low-quality and high-quality sellers. It appears that the expected payoff functions are very flat around 90, and in all treatments the profit at a cutoff level of 90 was close to the profit of the optimal cutoff level. This means that there was hardly any pressure to change the cutoff level. 
For sellers, we divided a group's social history screen of 40 observations in 8 windows of 5 observations each. The history screen of the sellers was ordered on signal cost (cf. Figure 2b), so the first window contained the observations related to the 1-5 highest signal costs, the second window contained the observations related to the 6-10 highest signal costs etc. Then we computed for each window how much profit the seller would have made on average, given the own type in the new period, if she had chosen the signal costs in the window and if she had faced the corresponding buy/not buy decisions of the buyers. Then we determined which window would have led to the highest average profit, and we set the behavioral response equal to the average signal cost in this window. Notice that the behavioral response of the sellers has the flavor of reinforcement and imitation. It circumvents the need to compute best responses altogether.

Table 9 presents sellers' signal costs combined with the best responses and the behavioral responses. In all treatments, the behavioral response comes closer to actual behavior than the best response does. In the treatments with noise behavioral responses were lower than best responses. This makes sense, because for the same signal cost, the observations in a window were ordered on signal. Higher signals were often accompanied by buy decisions. Thus, the highest hypothetical profit was often generated in windows with relatively low signal costs combined with lucky signals. In this way, reinforcement and imitation guided subjects to lower signal costs than the best response did. It is remarkable that in all treatments with noise, actual behavior was sandwiched between the behavioral response and the best response. Probably both the force of belief learning (best response) and the force of imitation/reinforcement (behavioral response) played a role for sellers.

\section{[ Insert Table 9 about here ]}

A noteworthy feature of sellers' behavior is that the volatility of the signal costs chosen by high-quality sellers increased with the noise in the signal. As the histograms presented in Figures 3 revealed, especially in treatment $\sigma_{120}$, highquality sellers chose signals all over the place when they attempted to separate. Figures 5a-5d provide a coherent reason why this may have occurred. These figures present the expected profit of a high-quality seller and its variance conditional on the signal cost submitted, given the actual average cutoff level of the buyers in a treatment. In treatment $\sigma_{0}$, the payoff function was steep and, indeed, highquality sellers' signal costs in this treatment were clustered in a very small interval. 
In treatment $\sigma_{120}$, the payoff function has become very flat. It is no surprise that high-quality sellers were not able to locate the optimum of this function.

\section{[ Insert Figures 5a-5d about here ]}

We now turn to an explanation of the two most important findings of our paper. The first is that subjects separated even in a treatment where the noise is so small that a pure strategy separating equilibrium did not exist. We already briefly alluded to an explanation of this intuitive anomaly. Buyers started with a natural cutoff level of approximately 90. In periods 21-40 of treatment $\sigma_{10}$, they actually earned on average 133.9 (at a standard error of 157.6). If they would consistently have used the much lower optimal best response cut-off level of $57.8,{ }^{24}$ their profit would have been 141.8 (standard error 166.0). In the large majority of $95 \%$ of the cases, the optimal cutoff led to the same choice and profit as the buyer actually made. In only $4 \%$ of the cases the optimal cutoff would have led to a higher profit, while in $1 \%$ of the cases it would have led to a lower profit. The difference between the actual profit and the optimal profit generated by a hypothetical cutoff strategy of 57.8 is not significant according to a Wilcoxon rank test using all observations as data-points $(p=0.15)$. Thus, there was no noticeable pressure on buyers to lower their cutoff. Given the high cutoff level actually used by the buyers, it is no surprise that high-quality sellers continued to send messages with high signal costs. In fact, their messages were even a bit lower than the actual best response (112.8 versus 117.9). So buyers employed higher cutoff levels than they should in equilibrium, but they were hardly punished for doing so and sellers were very close to best responding.

Finally, we deal with the issue of equilibrium selection. In the introduction, we hypothesized that sellers are less willing to pursue the separating equilibrium when the noise increases. With noise, there is always a chance that a separating signal cost of a high-quality seller is pushed below the cutoff of the buyer, in which case the seller incurs a loss. This becomes more likely the higher the noise is. For instance, in $\sigma_{40}$, the probability that the (equilibrium) separating signal cost of the high-quality seller is not accepted equals 0.05 . In $\sigma_{120}$, the probability that a high-quality seller incurs a loss increases to 0.23. At the same time, the equilibrium markup in case of a sale decreases from 258 in $\sigma_{40}$ to 169 in $\sigma_{120}$.

\footnotetext{
${ }^{24}$ If buyers would have used this cutoff level of $z^{B R}=57.8$, the theoretical best response for the bad (good) type seller is to choose signal costs equal to zero (81.34). So even in that case the bad type would not have an incentive to deviate from 0, i.e. to upset the separating outcome.
} 
Thus, for the seller the prospects of the separating equilibrium deteriorate when the noise in the signal increases.

The actual profit data are in accordance with this explanation. Table 10 lists seller profits, separated for high types and low types, and for pooling signals $(<90)$ and separating signals $(\geq 90)$. For high-quality types, the attractiveness of the separating equilibrium is highest in $\sigma_{0}$. With noise the loss-gain tradeoff worsens, which favors pooling. In fact, for $\sigma_{120}$ high-quality sellers made on average slightly more when they selected pooling signal costs. This result agrees with the finding that in the end 6 of the 8 matching-groups in $\sigma_{120}$ converged to pooling.

\section{[ Insert Table 10 about here ]}

\section{Conclusion}

In this paper we introduced noise in Spence's pure signaling game. Besides being more realistic, allowing for noise in the signal is appealing because it substantially cuts down the number of equilibria. With an unfavorable prior belief, the separating equilibrium even completely disappears for low levels of noise. It reappears for intermediate noise levels, where the (high type's) signal costs increase with the noise in the signal up to a ceiling. In contrast, a pooling (on no signaling) equilibrium always exists.

In our experiment, noise worked as an empirical (equilibrium) selection device. With low and medium noise, subjects successfully coordinated on separation. For high noise levels, the separating equilibrium lost ground to the pooling equilibrium. With high noise, high-quality sellers faced the risk that a signal cost aimed at separation would fail to accomplish its goal because it received a bad draw for the noise term. In addition, with high noise higher signal costs were required to convince the buyer, which decreased the markup in case of a sale. Thus, the separating equilibrium became much less attractive, which is reflected in the frequency that it was chosen. We did, however, observe a couple of very high signal costs when the noise in the signal cost was high.

We observed a smoother pattern in the effect of noise on the signal cost than predicted by theory. Conditional on choosing a separating level of signal costs, the signal costs of high-quality sellers increased monotonically with noise. This is surprising, because it means that subjects separated even in the case where no separating equilibrium existed. It turns out that with little noise subjects initially played as if there were no noise. In particular, buyers used higher cutoffs than 
prescribed by equilibrium. The strategic nature of the game was such that there was negligible pressure on buyers to change their initial behavior. Buyers almost made the same amount as they would have earned with their best response and their best response hardly guided them to lower cutoffs. Sellers reacted by choosing best responses to buyers' actual cutoffs. Thus, separation did not unravel.

In fact, simple behavioral explanations organize the data very well. Buyers' actual play follows the predictions of belief learning closely. According to this explanation, buyers form beliefs on the basis of the available information of past play and best respond to this belief. Sellers' behavior shows traces of reinforcement learning as well as belief learning. That is, high quality sellers usually choose signal costs that are lower than their empirical best responses but higher than the levels that are reinforced. An important advantage of the behavioral explanations is that they take account of our finding that subjects separated in the treatment where the noise was so small that a separating equilibrium did not exist. Thus, they account for this intuitive anomaly.

\section{Appendix}

In this Appendix we formally derive the theoretical predictions discussed in Section 2. Recall that $z=m+\sigma \cdot \varepsilon$, with $z$ the signal observed by the buyer, $m$ the signal costs chosen by the seller and $\varepsilon$ a random variable with distribution $F$. With regard to $F$ we make the following three assumptions:

(F.1) $F$ is continuously differentiable, i.e. density $f$ is continuous;

(F.2) The density $f$ is strictly positive on the entire real line;

(F.3) The conditional density of $z$ given $m$ (denoted $g(z \mid m)$ ) satisfies the strict monotone likelihood ratio property (MLRP): $\frac{g(z \mid m)}{g\left(z \mid m^{\prime}\right)}=\frac{\frac{1}{\sigma} \cdot f\left(\frac{z-m}{\sigma}\right)}{\frac{1}{\sigma} \cdot f\left(\frac{z-m^{\prime}}{\sigma}\right)}$ is strictly increasing in $z$ for $m>m^{\prime}$.

These three assumptions facilitate the equilibrium analysis. First, in the setup of both Matthews and Mirman (1983) and Carlsson and Dasgupta (1997), MLRP implies that the receiver necessarily uses a cutoff strategy in equilibrium. Building on their theoretical analysis, Lemma 1 below reveals that essentially the same result applies in our model where signaling constitutes a pure social waste. Second, the three assumptions together also imply that $f$ is 'nicely' shaped, see our Lemma 2 . 
Lemma 1. Let $\mu_{b}(m)\left[\mu_{g}(m)\right]$ denote the probability with which the bad [good] type seller chooses signal costs $m$ in equilibrium. Consider non-pooling equilibria only, i.e. $\mu_{b}(m) \neq \mu_{g}(m)$ for some $m \geq 0$. Assumptions (F.1) through (F.3) then imply that the buyer's best response rule is of the following form (with $z^{*} \in(-\infty,+\infty)$ ):

$$
\begin{aligned}
\pi(z) & =0 \text { if } z<z^{*} \\
& =1 \text { if } z>z^{*}
\end{aligned}
$$

with $\pi(z)$ the probability that the buyer 'Buys' after observing signal $z$.

Proof. To prove the lemma, we first show that the seller's equilibrium strategy is weakly monotonic in his type. Using this we subsequently show that the buyer's equilibrium payoff from buying is monotonically increasing in the observed signal, positive for large signals and negative for small signals. ${ }^{25}$

Let $p(m \mid \pi(z))=\int \pi(z) \cdot \frac{1}{\sigma} \cdot f\left(\frac{z-m}{\sigma}\right) d z$ denote the probability with which the buyer buys, given that she uses strategy $\pi(z)$ and the seller chooses signal cost $m$. For the type $t \in\{b, g\}$ seller, expected payoffs then equal $t \cdot p(m \mid \pi(z))-m$. Let $M_{t}$ be the set of maximizers of this expected payoff function. This set is non-empty because $f$, and thus $p(m \mid \pi(z))$, is continuous and the relevant range $[0, t]$ of signal costs $m$ is compact. Suppose there exists a $m_{b}^{\prime} \in M_{b}$ with $m_{b}^{\prime}>0$. It then holds that $g \cdot\left[p\left(m_{b}^{\prime} \mid \pi(z)\right)-p(m \mid \pi(z))\right]>b \cdot\left[p\left(m_{b}^{\prime} \mid \pi(z)\right)-p(m \mid \pi(z))\right] \geq m_{b}^{\prime}-m$ for all $m \in\left[0, m_{b}^{\prime}\right)$. The second inequality directly follows from $m_{b}^{\prime} \in M_{b}$ whereas the first follows from $g>b$. Thus, the good type strictly prefers $m_{b}^{\prime}>0$ over any lower level of signal costs. Because this holds for any $m_{b}^{\prime}>0$ in $M_{b}$, there exists a cutoff level $m^{c} \geq 0$ such that the bad (good) type necessarily chooses $m \leq m^{c}\left(m \geq m^{c}\right)$ in equilibrium. The seller's equilibrium strategy is thus weakly monotonic.

The buyer's expected payoffs of buying when she observes signal $z$ and the seller plays $\left(\mu_{b}, \mu_{g}\right)$ equal $V\left(z \mid\left(\mu_{b}, \mu_{g}\right)\right)=-y+[x+y] \cdot \beta(z)$. Here $\beta(z)$ denotes the buyer's posterior belief that the seller is of the good type after observing signal $z$ and given that the seller uses strategy $\left(\mu_{b}, \mu_{g}\right)$. By Assumption (F.2) this belief is determined by Bayes' rule everywhere:

$$
\begin{aligned}
\beta(z) & \equiv \operatorname{Pr}\left(\left(t=\text { good } \mid z,\left(\mu_{b}, \mu_{g}\right)\right)\right. \\
& =\frac{p \cdot \int f\left(\frac{z-m}{\sigma}\right) \cdot \mu_{g}(m) d m}{p \cdot \int f\left(\frac{z-m}{\sigma}\right) \cdot \mu_{g}(m) d m+(1-p) \cdot \int f\left(\frac{z-m}{\sigma}\right) \cdot \mu_{b}(m) d m}
\end{aligned}
$$

\footnotetext{
${ }^{25}$ The proof is in the spirit of Lemmas 1 and 2 in Matthews and Mirman (1983). Because they do not consider mixed strategies, however, our proof more closely follows the one of Proposition 3.1 in Carlsson and Dasgupta (1997).
} 
Given that $f$ is continuous it follows that $\beta(z)$ is continuous in $z$. Moreover, if $\mu_{b}(m) \neq \mu_{g}(m)$ for some $m \geq 0$, MLRP together with the weak monotonicity of the seller's strategy imply that $\beta(z)$ is strictly increasing in $z$ (cf. Milgrom, 1981, Proposition 2). ${ }^{26}$ This in turn implies that the buyer's expected payoffs $V\left(z \mid\left(\mu_{b}, \mu_{g}\right)\right)$ are continuous and strictly increasing in $z$. Suppose $V\left(z \mid\left(\mu_{b}, \mu_{g}\right)\right)>[<] 0$ for all $z$. Then the buyer always [never] buys irrespective of the value of $z$ and both seller types would strictly prefer $m=0$. This contradicts $\mu_{b} \neq \mu_{g}$. Hence there is a unique solution $z^{*}$ to $V\left(z \mid\left(\mu_{b}, \mu_{g}\right)\right)=0$.

Lemma 2. Assumptions (F.1) through (F.3) imply that $f(u)$ is uni-modal and strictly increasing [decreasing] in $u$ for $u<[>] M$, with $M$ denoting the mode.

Proof. Let $c_{1}<c_{3}$. We first show that $f(c)>\min \left\{f\left(c_{1}\right), f\left(c_{3}\right)\right\}$ for all $c \in\left(c_{1}, c_{3}\right)$. Suppose not. Then by the continuity of $f$ there exists a $c_{2} \in\left(c_{1}, c_{3}\right)$ for which $f\left(c_{2}\right) \leq f(c)$ for all $c \in\left[c_{1}, c_{3}\right]$ (i.e. $c_{2}$ is an interior global minimum of $f$ on the compact set $\left.\left[c_{1}, c_{3}\right]\right)$. Assumption (F.1) then also implies that there exists a $\Delta c \leq \min \left\{c_{3}-c_{2}, c_{2}-c_{1}\right\}$ (with $\Delta c>0$ ) such that $f\left(c_{2}-\Delta c\right) \geq f\left(c_{2}\right)$ and $f\left(c_{2}+\Delta c\right) \geq f\left(c_{2}\right)$. Pick $z_{h}$ and $m^{\prime}$ such that $z_{h}-m^{\prime}=\sigma\left(c_{2}+\Delta c\right)$, and take $z_{l}=\sigma c_{2}+m^{\prime}$ and $m=m^{\prime}+\sigma \Delta c$. Then $\frac{f\left(\frac{z_{h}-m}{\sigma}\right)}{f\left(\frac{z_{h}-m^{\prime}}{\sigma}\right)}=\frac{f\left(c_{2}\right)}{f\left(c_{2}+\Delta c\right)} \leq \frac{f\left(c_{2}-\Delta c\right)}{f\left(c_{2}\right)}=\frac{f\left(\frac{z_{l}-m}{\sigma}\right)}{f\left(\frac{z_{l}-m^{\prime}}{\sigma}\right)}$. This contradicts that $g(z \mid m)$ satisfies MLRP.

Given that $f$ is continuous and strictly positive on $\mathbb{R}$ it follows that $f$ cannot be monotonically increasing; otherwise $\int_{c_{1}}^{\infty} f(u) d u>\int_{c_{1}}^{\infty} f\left(c_{1}\right) d u=\infty$, contradicting that $f$ is a density. Together with $f(c)>\min \left\{f\left(c_{1}\right), f\left(c_{3}\right)\right\}$ for all $c \in\left(c_{1}, c_{3}\right)$ it follows that $f$ is uni-modal.

Assuming that players are restricted to use pure strategies only, Theorem 1 below characterizes the set of possible equilibria. Proposition 1 in the main text directly follows from this theorem.

${ }^{26}$ To see this directly, note that the sign of $\frac{\partial \beta(z)}{\partial z}$ equals the sign of $\frac{\partial l(z)}{\partial z}$, with $l(z)$ equal to:

$$
l(z) \equiv \frac{p \cdot \int f\left(\frac{z-m}{\sigma}\right) \cdot \mu_{g}(m) d m}{(1-p) \cdot \int f\left(\frac{z-m}{\sigma}\right) \cdot \mu_{b}(m) d m}=\frac{p \cdot \int\left[\frac{f\left(\frac{z-m}{\sigma}\right)}{f\left(\frac{z-m^{c}}{\sigma}\right)}\right] \cdot \mu_{g}(m) d m}{(1-p) \cdot \int\left[\frac{f\left(\frac{z-m}{\sigma}\right)}{f\left(\frac{z-m^{c}}{\sigma}\right)}\right] \cdot \mu_{b}(m) d m}
$$

The second equality simply follows from dividing both numerator and denominator by $f\left(\frac{z-m^{c}}{\sigma}\right)$, with $m^{c}$ defined in the main text. By MLRP, the numerator is strictly increasing in $z$ whenever there exists a $m>m^{c}$ for which $\mu_{g}(m)>0$. Similarly so, the denominator is strictly decreasing in $z$ if there exists a $m<m^{c}$ for which $\mu_{b}(m)>0$. Hence $l(z)$ is strictly increasing in $z$ whenever $\mu_{b} \neq \mu_{g}$. 
Theorem 1. Assume that players are restricted to use pure strategies. (i) A pooling equilibrium in which both seller types choose $m=0$ always exists. In this equilibrium the buyer never [always] buys when $p<[>] \beta^{*} \equiv \frac{y}{x+y}$. Pooling on some $m>0$ cannot occur. (ii) Generically, i.e. for all $p \neq \frac{\beta^{*} \cdot g}{\left(1-\beta^{*}\right) \cdot b+\beta^{*} \cdot g}$, it holds that in any separating equilibrium the bad type seller chooses $m=0$ whereas the good type chooses some positive level of signal costs $m=m_{g}>0$. The buyer buys if $z>z^{*}$ and refrains from buying otherwise. Necessary and sufficient conditions for $\left(\left(0, m_{g}\right) ; z^{*}\right)$ to constitute equilibrium strategies are:

$$
\begin{gathered}
\begin{array}{c}
\frac{g}{\sigma} \cdot f\left(\frac{z^{*}-m_{g}}{\sigma}\right)=1 \text { with } m_{g}>z^{*}-\sigma M \\
\frac{1}{\sigma} \cdot f\left(\frac{z^{*}}{\sigma}\right)=\frac{p \cdot\left(1-\beta^{*}\right)}{\beta^{*} \cdot(1-p)} \cdot \frac{1}{g} \\
b \cdot\left(F\left(\frac{z^{*}}{\sigma}\right)-F\left(\frac{z^{*}-m_{b}}{\sigma}\right)\right) \leq m_{b} \\
\text { for } m_{b}>z^{*}-\sigma M \text { that solves } \frac{b}{\sigma} \cdot f\left(\frac{z^{*}-m_{b}}{\sigma}\right)=1 \\
g \cdot\left(F\left(\frac{z^{*}}{\sigma}\right)-F\left(\frac{z^{*}-m_{g}}{\sigma}\right)\right) \geq m_{g}
\end{array}
\end{gathered}
$$

Proof. (i) For any pooling strategy it follows from $(A 2)$ that the buyer's posterior belief equals her prior belief for any signal $z$ observed; $\beta(z)=p \forall z$. Therefore, if $p \cdot x-(1-p) \cdot y>0$, i.e. $p>\beta^{*} \equiv \frac{y}{x+y}$, the buyer buys for sure and neither type of seller wants to spend positive signaling costs. Similarly, in case $p<\beta^{*}$ the buyer never buys and the seller's unique best response is $m=0$. Only in the knife-edge case $p=\beta^{*}$ the buyer is indifferent and her probability of buying $\pi(z)$ may vary with $z$. For the type $t$ seller (with $t \in\{b, g\}$ ) to be willing to choose some $m>0$ it must then hold that $t \cdot \frac{\partial p(m \mid \pi(z))}{\partial m}=1$, where $p(m \mid \pi(z))=\int \pi(z) \cdot \frac{1}{\sigma} \cdot f\left(\frac{z-m}{\sigma}\right) d z$ denotes the probability of trade, given buyer's strategy $\pi(z)$ and the seller choosing signal costs $m .{ }^{27}$ With $b<g$ this first order condition cannot hold simultaneously for both types, so pooling on some $m>0$ cannot occur.

(ii) From Lemma 1 it follows that in any non-pooling equilibrium the buyer necessarily uses a cutoff strategy like in $(A 1)$. The expected payoff of choosing

\footnotetext{
${ }^{27}$ By Assumption (F.1) $\frac{\partial p(m \mid \pi(z))}{\partial m}$ does exist. In fact, $\frac{\partial p(m \mid \pi(z))}{\partial m}=\int \frac{1}{\sigma} \cdot f\left(\frac{z-m}{\sigma}\right) d \pi(z)$, see Carlsson and Dasgupta (1997, fn. 8).
} 
$m$ for the type $t$ seller then equals $t \cdot\left(1-F\left(\frac{z^{*}-m}{\sigma}\right)\right)-m$. Hence the following necessary first order condition for an interior maximum:

$$
\frac{t}{\sigma} \cdot f\left(\frac{z^{*}-m}{\sigma}\right)=1
$$

The l.h.s. (r.h.s) equals the marginal benefits (costs) of raising $m$. With $b<g$, for given $m$ condition $(A 7)$ cannot hold simultaneously for both types. The two seller types thus never put positive probability on the same $m>0$ in equilibrium.

From Lemma 2 it follows that $(A 7)$ allows at most two solutions. If so, only the largest one satisfies the second order condition, because at the optimum $f$ should be increasing. (If $f$ is decreasing, an increase in $m$ at the margin increases the marginal benefits of raising $m$ further.) Therefore, necessarily $\frac{z^{*}-m_{t}}{\sigma}<M$. In equilibrium the type $t$ seller thus chooses between the two levels $m=0$ and $m_{t}>z^{*}-\sigma M$ satisfying $(A 7)$ only.

We next show that the bad type seller necessarily chooses $m=0$. Suppose to the contrary that he chooses $m_{b}>0$. This requires that $m_{b}$ yields the bad type weakly more than $m=0$ does. Given $g>b$ the good type then already strictly earns more by choosing $m_{b}$ rather than $m=0$, so certainly this is the case for signal costs equal to $m_{g}$. Hence for all levels of signal costs chosen in equilibrium first order condition $(A 7)$ holds. From Bayes' rule in $(A 2)$ we then obtain that after observing cutoff signal $z^{*}$, the buyer's posterior belief equals:

$$
\beta\left(z^{*}\right)=\frac{p \cdot \frac{1}{g}}{p \cdot \frac{1}{g}+(1-p) \cdot \frac{1}{b}}=\frac{p \cdot b}{p \cdot b+(1-p) \cdot g}
$$

Because $\beta(z)$ is continuous in $z$, it necessarily must be such that the buyer is indifferent between her two actions after observing cutoff signal $z^{*}$ (cf. Carlsson and Dasgupta, 1997):

$$
\beta\left(z^{*}\right)=\beta^{*} \equiv \frac{y}{x+y}
$$

Since generically $\frac{p \cdot b}{p \cdot b+(1-p) \cdot g} \neq \frac{y}{x+y}$, an equilibrium in which neither type chooses $m=0$ cannot exist. Therefore, the bad type seller necessarily chooses $m=0$.

Only two possible types of pure strategy equilibria remain: (1) both seller types choose $m=0$ (cf. case (i)), and (2) the bad type chooses $m=0$ whereas the good type chooses $m_{g}>0$ satisfying $(A 3)$. Consider the latter case. From $(A 2)$ and $(A 3)$ we obtain that:

$$
\beta\left(z^{*}\right)=\frac{p}{p+(1-p) \cdot g \cdot \frac{1}{\sigma} \cdot f\left(\frac{z^{*}}{\sigma}\right)}
$$


Together with requirement $(A 8)$ then equality $(A 4)$ follows. Note that generically, this latter equality has either two, or zero solutions (cf. Lemma 2).

Given cutoff $z^{*}$ implicitly defined in $(A 4)$, the bad type seller should not have an incentive to deviate from $m=0$. The best candidate deviation level of signal costs $m_{b}$ necessarily satisfies first order condition $(A 7)$ and $m_{b}>z^{*}-\sigma M$ as well. This level $m_{b}$ should give the bad type seller weakly less than choosing $m=0$, i.e.:

$$
b \cdot\left(1-F\left(\frac{z^{*}}{\sigma}\right)\right) \geq b \cdot\left(1-F\left(\frac{z^{*}-m_{b}}{\sigma}\right)\right)-m_{b}
$$

Rewriting this yields requirement $(A 5)$. (From Lemma 2 it follows that only when $\frac{b}{\sigma} \cdot f\left(\frac{z^{*}}{\sigma}\right)<1$ no $m_{b}>z^{*}-\sigma M$ exists that solves the first order condition $(A 7)$; in that case condition ( $A 5)$ is automatically satisfied.) Similarly so, the good type seller should (weakly) prefer choosing $m_{g}>0$ over no signal costs at all. This is what condition $(A 6)$ requires.

The actual existence of a separating equilibrium depends on whether expressions (A3) through $(A 6)$ in Theorem 1 allow a feasible solution. Theorem 2 below, which generalizes Proposition 2 in the main text to general distribution functions $F$, in particular considers how this varies with the value of $\sigma$. For the situation in which the level of noise becomes small, the following lemma will appear helpful in proving this theorem. In words it says that the tails of density $f(u)$ become 'thin' if we move sufficiently far away from the mode $M$ (see part (a)). As a consequence, we should stay sufficiently close to the mode $M$ if we want $f(u)$ to equal a particular given value $v$ (cf. part (b)).

Lemma 3. Assumptions (F.1) through (F.3) imply that:

(a) $\forall_{k>0} \exists_{\bar{U}(k)>0}$ such that $f(|u|) \leq \frac{k}{|u|}$ for all $|u| \geq \bar{U}(k)$;

(b) Let $f_{+}^{-1}\left(f_{-}^{-1}\right)$ denote the inverse of $f(u)$ on the interval $u \geq M(u \leq M)$, with $M$ the mode of $f(u) .{ }^{28}$ It holds that: $\forall_{k>0} \exists_{\underline{V}(k)>0}$ such that $-\frac{k}{v} \leq$ $f_{-}^{-1}(v) \leq M \leq f_{+}^{-1}(v) \leq \frac{k}{v}$ for all $v$ satisfying $0<v \leq \underline{V}(k)$.

Proof. (a) First consider the case $u>0$. Let $D \equiv\left\{u \mid f(u)=\frac{k}{u}\right\}$ denote the set of intersection points of $f(u)$ and $h(u ; k) \equiv \frac{k}{u}$. First suppose that this set is bounded (which includes the case that $D$ is empty), i.e. $\exists_{U(k)>0}$ such that

\footnotetext{
${ }^{28}$ Given that $f$ is monotonically increasing below $M$ and monotonically decreasing above $M$ (cf. Lemma 2), these inverses do exist.
} 
$u \leq U(k)$ for all $u \in D$. Then by the continuity of $f$ and $h(u ; k)$ on $(0, \infty)$, for all $u>U(k)$ either $f(u)>\frac{k}{u}$ or $f(u)<\frac{k}{u}$. Now the former would imply:

$$
\int_{U(k)}^{\infty} f(u) d u>\int_{U(k)}^{\infty} \frac{k}{u} d u=k \cdot\left(\lim _{u \longrightarrow \infty} \ln u-\ln U(k)\right)=\infty .
$$

This contradicts $\int_{U(k)}^{\infty} f(u) d u \leq 1$ given that $f$ is a density. Hence it must hold that $f(u)<\frac{k}{u}$ for all $u>U(k)$.

Next assume that the set of intersection points $D$ is unbounded. Let $u_{1}, u_{3} \in D$ with $u_{1}<u_{3}$ and $f(u)<\frac{k}{u}$ for all $u$ satisfying $u_{1}<u<u_{3}$. Such $u_{1}$ and $u_{3}$ do exist when $D$ is unbounded, because otherwise $f(u) \geq \frac{k}{u}$ for all $u \geq u_{1}$ and $\int_{u_{1}}^{\infty} f(u) d u=\infty$, a contradiction. Define $u_{2} \equiv \frac{u_{1}+u_{3}}{2}$. MLRP then requires $\frac{f\left(u_{1}\right)}{f\left(u_{2}\right)}<\frac{f\left(u_{2}\right)}{f\left(u_{3}\right)}{ }^{29}$ Given $u_{1}, u_{3} \in D$ this becomes $\frac{k^{2}}{u_{1} \cdot u_{3}}<\left[f\left(u_{2}\right)\right]^{2}$. From $f\left(u_{2}\right)<\frac{k}{u_{2}}$ this can only be satisfied whenever $\frac{k^{2}}{u_{1} \cdot u_{3}}<\frac{k^{2}}{u_{2}^{2}}$, i.e. $\left(\frac{u_{1}+u_{3}}{2}\right)^{2}<u_{1} \cdot u_{3}$. Rewriting this we get $\left(u_{1}-u_{3}\right)^{2}<0$, a contradiction. Hence $D$ cannot be unbounded.

The case $u<0$ is simply the mirror image of $u>0$ and thus immediately follows from the above.

(b) This part follows from part (a). To see this, consider the case $u \geq M$. Here $f$ - and thus $f_{+}^{-1}$ - is decreasing. Let $\bar{U}(k)>0$ be the cutoff value as given in part (a), i.e. $f(u) \leq \frac{k}{u}$ for all $u \geq \bar{U}(k)$. Consider values $v \leq f(\bar{U}(k)) \equiv \underline{V}(k)$. From $f_{+}^{-1}$ decreasing it follows that for all these values $f_{+}^{-1}(v) \geq f_{+}^{-1}(f(\bar{U}(k)))=\bar{U}(k)$. Now suppose there exists a $v^{\prime}$ with $0<v^{\prime} \leq \underline{V}(k)$ for which $f_{+}^{-1}\left(v^{\prime}\right)>\frac{k}{v^{\prime}}$. Given that function $h(u ; k) \equiv \frac{k}{u}$ is strictly decreasing, it holds that $h\left(f_{+}^{-1}\left(v^{\prime}\right) ; k\right)<$ $h\left(\frac{k}{v^{\prime}} ; k\right)=v^{\prime}=f\left(f_{+}^{-1}\left(v^{\prime}\right)\right)$. So, at point $f_{+}^{-1}\left(v^{\prime}\right)$ function $f(u)$ lies above function $h(u ; k)$. Together with $f_{+}^{-1}\left(v^{\prime}\right) \geq \bar{U}(k)$ this contradicts part (a). Hence necessarily $f_{+}^{-1}(v) \leq \frac{k}{v}$ for all $v$ satisfying $0<v \leq \underline{V}(k)$. (Note that $M \leq f_{+}^{-1}(v)$ follows by definition.) Again, the case $u \leq M$ is the mirror image of $u \geq M$.

Theorem 2. Assume that players are restricted to use pure strategies. A necessary condition for a separating equilibrium to exist is that $\sigma \leq g \cdot f(M)$. $\min \left\{\frac{(1-p) \beta^{*}}{p\left(1-\beta^{*}\right)}, 1\right\} \equiv \bar{\sigma}$. Assuming $\sigma \leq \bar{\sigma}$, it holds that:

(i) $p \leq \beta^{*}$ : a separating equilibrium does not exist if $\sigma$ becomes sufficiently small;

(ii) $\beta^{*}<p<\frac{\beta^{*} \cdot g}{\left(1-\beta^{*}\right) \cdot b+\beta^{*} \cdot g}$ : a separating equilibrium always exists. For this equilibrium it holds that $\lim _{\sigma \downarrow 0} m_{g}=0$;

\footnotetext{
${ }^{29}$ To see this, pick $z_{h}$ and $m^{\prime}$ such that $z_{h}-m^{\prime}=\sigma u_{3}$. Then let $z_{l}=\sigma u_{2}+m^{\prime}$ and $m=$ $m^{\prime}+\sigma\left(u_{2}-u_{1}\right)$. This gives $\frac{z_{h}-m^{\prime}}{\sigma}=u_{3}, \frac{z_{h}-m}{\sigma}=u_{2}=\frac{z_{l}-m^{\prime}}{\sigma}$ and $\frac{z_{l}-m}{\sigma}=u_{1}$. The requirement then follows from MLRP.
} 
(iii) $p>\frac{\beta^{*} \cdot g}{\left(1-\beta^{*}\right) \cdot b+\beta^{*} \cdot g}$ : a separating equilibrium does not exist.

Proof. When $\sigma>\bar{\sigma}$ either $(A 3)$ or $(A 4)$ in Theorem 1 does not have a solution, so a separating equilibrium cannot exist. Therefore, $\sigma \leq \bar{\sigma}$ is a necessary condition.

Before proving (i) through (iii) separately, we first show that $\lim _{\sigma \downarrow 0} z^{*}=$ $\lim _{\sigma \downarrow 0} m_{g}=\lim _{\sigma \downarrow 0} m_{b}=0$ (with $m_{b}$ the solution to $(A 7)$ for $t=b$ ). Consider the defining equation $(A 4)$ of $z^{*}$ and let $c=\frac{p \cdot\left(1-\beta^{*}\right)}{\beta^{*} \cdot(1-p)} \cdot \frac{1}{g}$. From Lemma 2 it follows that (generically) this equation has either two or no solutions. For $\sigma$ low enough, (A4) admits two solutions. Denote these solutions $z_{l}^{*}$ and $z_{h}^{*}$ respectively, with $z_{l}^{*}<z_{h}^{*}$. Note that necessarily $\frac{z_{l}^{*}}{\sigma}<M$ and $\frac{z_{h}^{*}}{\sigma}>M$. First consider the latter solution $z_{h}^{*}$. With $f_{+}^{-1}$ denoting the inverse of $f$ on the interval above $M$, we obtain $z_{h}^{*}=\sigma \cdot f_{+}^{-1}(\sigma c)$ from $(A 4)$. From Lemma 3(b) it then follows that for all $\sigma$ satisfying $0<\sigma \leq \frac{\underline{V}(k)}{c} \equiv \bar{\sigma}(k ; c)$, necessarily $f_{+}^{-1}(\sigma c) \leq \frac{k}{\sigma c}$. Hence $z_{h}^{*}=$ $\sigma \cdot f_{+}^{-1}(\sigma c) \leq \sigma \cdot \frac{k}{\sigma c}=\frac{k}{c}$ for all $\sigma \leq \bar{\sigma}(k ; c)$. Because this holds for any arbitrary $k>0$, and $z_{h}^{*}>\sigma M$, we obtain $\lim _{\sigma \downarrow 0} z_{h}^{*}=0$.

Next consider a solution $z_{l}^{*}$ to $(A 4)$ for which $\frac{z_{l}^{*}}{\sigma}<M$. In this case $z_{l}^{*}=$ $\sigma \cdot f_{-}^{-1}(\sigma c)$ from $(A 4)$, with $f_{-}^{-1}$ the inverse of $f$ on the interval below $M$. From Lemma 3(b) it then follows that for all $\sigma \leq \frac{\underline{V}(k)}{c} \equiv \bar{\sigma}(k ; c)$, necessarily $f_{-}^{-1}(\sigma c) \geq$ $-\frac{k}{\sigma c}$. Hence $z_{l}^{*}=\sigma \cdot f_{-}^{-1}(\sigma c) \geq-\frac{k}{c}$ for all $\sigma \leq \bar{\sigma}(k ; c)$. Because this holds for any arbitrary $k>0$, and $z_{l}^{*}<\sigma M$, we obtain $\lim _{\sigma \downarrow 0} z_{l}^{*}=0$. Hence, overall $\lim _{\sigma \downarrow 0}$ $z^{*}=0$ for any solution $z^{*}$ to $(A 4)$.

By inserting $z_{l}^{*}=z^{*}-m_{g}$ and $c=\frac{1}{g}$ in the reasoning for $z_{l}^{*}$ above we immediately obtain $\lim _{\sigma \downarrow 0}\left(z^{*}-m_{g}\right)=0$ from equation $(A 3)$. Together with $\lim _{\sigma \downarrow 0}$ $z^{*}=0$ this implies $\lim _{\sigma \downarrow 0} m_{g}=0$. Similarly so for $\lim _{\sigma \downarrow 0} m_{b}=0$.

(i). Inequality $p \leq \beta^{*}$ is equivalent to $\frac{p \cdot\left(1-\beta^{*}\right)}{\beta^{*} \cdot(1-p)} \cdot \frac{1}{g} \leq \frac{1}{g}$. From conditions $(A 3)$ and $(A 4)$ in Theorem 1 we obtain that $f\left(\frac{z^{*}-m_{g}}{\sigma}\right) \geq f\left(\frac{z^{*}}{\sigma}\right)$ necessarily. Together with Lemma 2 this implies that necessarily $\frac{z^{*}}{\sigma}>M$. Hence in this case only a separating equilibrium based on $z_{h}^{*}$ may exist. We show that this separating equilibrium disappears for $\sigma$ sufficiently small, because the bad type seller obtains an incentive to deviate from $m=0$ to a positive level of signal costs equal to $m_{b}$. To see this, from equality $(A 4)$ we have that $\lim _{\sigma \downarrow 0} f\left(\frac{z_{h}^{*}}{\sigma}\right)=0$. With Lemma 2 and $\frac{z_{h}^{*}}{\sigma}>M$ this in turn implies that $\lim _{\sigma \downarrow 0} \frac{z_{h}^{*}}{\sigma}=\infty$. Taking the limit in the l.h.s. of condition $(A 5)$ we then obtain that:

$$
\lim _{\sigma \downarrow 0} b \cdot\left(F\left(\frac{z_{h}^{*}}{\sigma}\right)-F\left(\frac{z_{h}^{*}-m_{b}}{\sigma}\right)\right)=b>0
$$


Here $\lim _{\sigma \downarrow 0} \frac{z_{h}^{*}-m_{b}}{\sigma}=-\infty$ (and thus $\lim _{\sigma \downarrow 0} F\left(\frac{z_{h}^{*}-m_{b}}{\sigma}\right)=0$ ) follows from the fact that $m_{b}$ satisfies both first order condition $(A 7)$ and $m_{b}>z_{h}^{*}-\sigma M$ (such that $\left.\frac{z_{h}^{*}-m_{b}}{\sigma}<M\right)$. Because $\lim _{\sigma \downarrow 0} m_{b}=0$ as derived above, requirement $(A 5)$ cannot be satisfied for $\sigma$ sufficiently small.

(ii). When $\beta^{*}<p<\frac{\beta^{*} \cdot g}{\left(1-\beta^{*}\right) \cdot b+\beta^{*} \cdot g}$ separating equilibria based on $z_{l}^{*}$ and $z_{h}^{*}$ may exist side by side. The one based on $z_{h}^{*}$ vanishes for low $\sigma$, see the proof of part (i). We show that the one based on $z_{l}^{*}$ continues to exist under the stated conditions. Note that when $\sigma \leq \bar{\sigma}$, condition $(A 4)$ allows a solution $z_{l}^{*} \leq \sigma M$. Given this solution $z_{l}^{*}$ and $\frac{p\left(1-\beta^{*}\right)}{(1-p) \beta^{*}}>1$ from $\beta^{*}<p$, we can always find a corresponding solution $m_{g}$ to $(A 3)$. Because $p<\frac{\beta^{*} \cdot g}{\left(1-\beta^{*}\right) \cdot b+\beta^{*} \cdot g}$, it follows from $(A 4)$ that $\frac{b}{\sigma}$. $f\left(\frac{z_{l}^{*}}{\sigma}\right)=\frac{b}{g} \cdot \frac{p\left(1-\beta^{*}\right)}{(1-p) \beta^{*}}<1$. This implies that condition $(A 5)$ is automatically satisfied, because no $m_{b}>z^{*}-\sigma M$ exists that satisfies the first order condition $(A 7)$ (see the proof of Theorem 1). Moreover, from (A3) and Lemma 2 we have:

$$
\begin{gathered}
g \cdot\left(F\left(\frac{z_{l}^{*}}{\sigma}\right)-F\right. \\
\left.\left(\frac{z_{l}^{*}-m_{g}}{\sigma}\right)\right)=g \cdot \int_{z_{l}^{*}-m_{g}}^{z_{l}^{*}} \frac{1}{\sigma} \cdot f\left(\frac{u}{\sigma}\right) d u \\
>g \cdot \int_{z_{l}^{*}-m_{g}}^{z_{l}^{*}} \frac{1}{g} d u=m_{g}
\end{gathered}
$$

Hence condition $(A 6)$ is satisfied as well.

(iii). In case $p>\frac{\beta^{*} \cdot g}{\left(1-\beta^{*}\right) \cdot b+\beta^{*} \cdot g},(A 4)$ implies that $\frac{b}{\sigma} \cdot f\left(\frac{z^{*}}{\sigma}\right)=\frac{b}{g} \cdot \frac{p\left(1-\beta^{*}\right)}{(1-p) \beta^{*}}>1$. At $m=0$ the marginal benefits for the bad type of raising the signal costs thus exceed the marginal costs of doing so and he wants to deviate from choosing $m=0$. That is, condition $(A 5)$ cannot be satisfied.

The non-existence of a separating equilibrium in cases (i) and (iii) is based on the same intuition. Equation $(A 4)$ in Theorem 1 provides a precise characterization of the buyer's equilibrium cutoff value $z^{*}$ on the basis of her posterior beliefs. But the feasible value(s) of $z^{*}$ may be incompatible with seller's best response behavior given the buyer's cutoff strategy. In particular, no-deviation condition $(A 5)$ for the bad type seller may not be satisfied for the value(s) of $z^{*}$ that solve $(A 4)$. He thus obtains an incentive to deviate from $m=0$.

We finally turn to mixed strategy equilibria. Theorem 3 below characterizes the set of mixed strategy equilibria that potentially may exist.

Theorem 3. When players are allowed to use mixed strategies, only two additional types of (mixed strategy) equilibria may potentially exist: 
(i) The bad type chooses $m=0$ with probability $1-q_{b}$ and some $m_{b}>0$ with probability $q_{b}$, whereas the good type chooses some $m_{g} \geq m_{b}$ for sure. $A$ necessary condition for existence is: $p<\frac{\beta^{*} \cdot g}{\left(1-\beta^{*}\right) \cdot b+\beta^{*} \cdot g}$. Conditional on existence it holds that: $\lim _{\sigma \downarrow 0} z^{*}=\lim _{\sigma \downarrow 0} m_{b}=\lim _{\sigma \downarrow 0} m_{g}=b$ and $\lim _{\sigma \downarrow 0}$ $q_{b}=\frac{\left(1-\beta^{*}\right) \cdot p}{(1-p) \cdot \beta^{*}} \cdot \frac{b}{g} \equiv q_{0}$.

(ii) The bad type chooses $m=0$ for sure while the good type chooses $m=0$ with probability $1-q_{g}$ and some $m_{g}>0$ with probability $q_{g}$. A necessary condition for existence is: $p<\beta^{*}$. Conditional on existence it holds that: $\lim _{\sigma \downarrow 0} z^{*}=\lim _{\sigma \downarrow 0} m_{g}=g$ and $\lim _{\sigma \downarrow 0} q_{g}=0$.

Proof. From the proof of Theorem 1 we obtain the following three observations: (1) in equilibrium the type $t$ seller chooses between the two levels of signal costs $m=0$ and $m_{t}>z^{*}-\sigma M$ satisfying $(A 7)$ only, (2) the bad type seller necessarily puts positive probability on $m=0$, and (3) if the bad type puts positive probability on $m_{b}>0$ as well, the good type strictly prefers level $m_{g} \geq m_{b}$ over $m=0$ and thus chooses $m_{g}$ for sure. Together these three observations imply that only mixed strategy equilibria of types (i) and (ii) may potentially exist. The remainder of the proof characterizes these mixed equilibria in more detail and considers the limit equilibria of letting $\sigma$ become infinitely small.

First note that in a mixed equilibrium it necessarily holds that $z^{*}>\sigma M$. Suppose $z^{*} \leq \sigma M$. Then $f\left(\frac{z^{*}-m}{\sigma}\right)<f\left(\frac{z^{*}}{\sigma}\right)$ by Lemma 2 and neither type wants to mix between 0 and $m$ (at $m$ marginal benefits equal marginal costs, so at all inframarginal levels below $m$ marginal benefits exceed marginal costs).

(i) Equilibrium values of $z^{*}, m_{b}, m_{g}$ and $q_{b}$ are characterized by the following four equations:

$$
\begin{gathered}
\frac{b}{\sigma} \cdot f\left(\frac{z^{*}-m_{b}}{\sigma}\right)=1 \text { with } m_{b}>z^{*}-\sigma M \\
\frac{g}{\sigma} \cdot f\left(\frac{z^{*}-m_{g}}{\sigma}\right)=1 \text { with } m_{g}>z^{*}-\sigma M \\
\frac{b}{\sigma} \cdot f\left(\frac{z^{*}}{\sigma}\right)=\frac{\left(1-\beta^{*}\right) \cdot p \cdot b-\beta^{*} \cdot(1-p) \cdot g \cdot q_{b}}{\beta^{*} \cdot\left(1-q_{b}\right) \cdot(1-p) \cdot g} \text { with } z^{*}>\sigma M \\
b \cdot\left(F\left(\frac{z^{*}}{\sigma}\right)-F\left(\frac{z^{*}-m_{b}}{\sigma}\right)\right)=m_{b}
\end{gathered}
$$


Given the last condition, no-deviation requirement $(A 6)$ for the good type is automatically satisfied. Existence depends on whether $(A 9)$ through $(A 12)$ admit a feasible solution. Because these are four non-linear equations (in four unknowns), it is in general hard to determine whether a solution exists. We therefore look at the equilibrium properties when $\sigma$ becomes small, assuming the mixed strategy equilibrium to exist. The latter requires necessarily $\frac{\left(1-\beta^{*}\right) \cdot p \cdot b-\beta^{*} \cdot(1-p) \cdot g \cdot q_{b}}{\beta^{*} \cdot\left(1-q_{b}\right) \cdot(1-p) \cdot g}<1$, for otherwise $f\left(\frac{z^{*}-m_{b}}{\sigma}\right)<f\left(\frac{z^{*}}{\sigma}\right)$ and the bad type does not want to mix. This reduces to $p<\frac{\beta^{*} \cdot g}{\left(1-\beta^{*}\right) \cdot b+\beta^{*} \cdot g}$. Because $f>0$, from $(A 11)$ it follows that $q_{b}<\frac{\left(1-\beta^{*}\right) \cdot p}{(1-p) \cdot \beta^{*}} \cdot \frac{b}{g} \equiv q_{0}$ necessarily. (Note that for $p<\frac{\beta^{*} \cdot g}{\left(1-\beta^{*}\right) \cdot b+\beta^{*} \cdot g}$ it holds that $q_{0}<1$.)

From $(A 9)$ and $(A 10)$ it follows that $\lim _{\sigma \downarrow 0}\left(z^{*}-m_{b}\right)=\lim _{\sigma \downarrow 0}\left(z^{*}-m_{g}\right)=0$, see the proof of Theorem 2. Moreover, equality (A12) implies $\lim _{\sigma \downarrow 0} m_{b}=b$. Together, $\lim _{\sigma \downarrow 0} z^{*}=\lim _{\sigma \downarrow 0} m_{b}=\lim _{\sigma \downarrow 0} m_{g}=b$. Now suppose $\lim _{\sigma \downarrow 0} q_{b} \neq q_{0}$. Then there exists some $r<q_{0}$ such that $q_{b} \leq r$ for some subsequence $\sigma_{r} \downarrow 0$. Let $z_{r}$ solve $\frac{b}{\sigma} \cdot f\left(\frac{z_{r}}{\sigma}\right)=\frac{\left(1-\beta^{*}\right) \cdot p \cdot b-\beta^{*} \cdot(1-p) \cdot g \cdot r}{\beta^{*} \cdot(1-r) \cdot(1-p) \cdot g} \equiv k(r)$. From the proof of Theorem 2 it follows that $\lim _{\sigma \downarrow 0} z_{r}=0$. With $k(r)$ decreasing in $r$ (given $p<\frac{\beta^{*} \cdot g}{\left(1-\beta^{*}\right) \cdot b+\beta^{*} \cdot g}$ ) and $f\left(\frac{z}{\sigma}\right)$ decreasing in $z$ for $z>\sigma M$ (cf. Lemma 2), it follows that $z^{*} \leq z_{r}$ for all $\sigma_{r} \downarrow 0$, and thus $z^{*} \rightarrow 0$ along this subsequence. This contradicts $\lim _{\sigma \downarrow 0}$ $z^{*}=b>0$. Hence necessarily $\lim _{\sigma \downarrow 0} q_{b}=q_{0}$.

(ii) In this case equilibrium values of $z^{*}, m_{g}$ and $q_{g}$ are characterized by the following three equations:

$$
\begin{gathered}
\frac{g}{\sigma} \cdot f\left(\frac{z^{*}-m_{g}}{\sigma}\right)=1 \text { with } m_{g}>z^{*}-\sigma M \\
\frac{g}{\sigma} \cdot f\left(\frac{z^{*}}{\sigma}\right)=\frac{\left(1-\beta^{*}\right) \cdot p \cdot q_{g}}{\left(\beta^{*}-p\right)+\left(1-\beta^{*}\right) \cdot p \cdot q_{g}} \text { with } z^{*}>\sigma M \\
g \cdot\left(F\left(\frac{z^{*}}{\sigma}\right)-F\left(\frac{z^{*}-m_{g}}{\sigma}\right)\right)=m_{g}
\end{gathered}
$$

Given $(A 15)$, requirement $(A 5)$ for the bad type seller is satisfied. We again look at the equilibrium properties for low values of $\sigma$, assuming the mixed strategy equilibrium to exist. This requires $\frac{\left(1-\beta^{*}\right) \cdot p \cdot q_{g}}{\left(\beta^{*}-p\right)+\left(1-\beta^{*}\right) \cdot p \cdot q_{g}}<1$, for otherwise $f\left(\frac{z^{*}-m_{g}}{\sigma}\right)<$ $f\left(\frac{z^{*}}{\sigma}\right)$ and the good type does not want to mix. Therefore $p<\beta^{*}$ is needed.

From $(A 13)$ it follows that $\lim _{\sigma \downarrow 0} \frac{z^{*}-m_{g}}{\sigma}=-\infty$ and $\lim _{\sigma \downarrow 0}\left(z^{*}-m_{g}\right)=0$, see the proof of Theorem 2. Because the r.h.s. of $(A 14)$ is bounded from above by $\frac{\left(1-\beta^{*}\right) \cdot p}{(1-p) \cdot \beta^{*}}$, the l.h.s. is bounded as well. This implies $\lim _{\sigma \downarrow 0} f\left(\frac{z^{*}}{\sigma}\right)=0$ and thus $\lim _{\sigma \downarrow 0} \frac{z^{*}}{\sigma}=\infty$. Together with equality $(A 15)$ we obtain $\lim _{\sigma \downarrow 0} m_{g}=g$, and thus $\lim _{\sigma \downarrow 0} z^{*}=g$ as well. Now suppose $\lim _{\sigma \downarrow 0} q_{g} \neq 0$. Then there exists some 
$r>0$ such that $q_{g} \geq r$ for some subsequence $\sigma_{r} \downarrow 0$. Let $z_{r}$ solve $\frac{g}{\sigma} \cdot f\left(\frac{z_{r}}{\sigma}\right)=$ $\frac{\left(1-\beta^{*}\right) \cdot p \cdot r}{\left(\beta^{*}-p\right)+\left(1-\beta^{*}\right) \cdot p \cdot r} \equiv l(r)$. From the proof of Theorem 2 it follows that $\lim _{\sigma \downarrow 0} z_{r}=0$. With $l(r)$ increasing in $r$ and $f\left(\frac{z}{\sigma}\right)$ decreasing in $z$ for $z>\sigma M$ (cf. Lemma 2), it follows that $z^{*} \leq z_{r}$ for all $\sigma_{r} \downarrow 0$, and thus $z^{*} \rightarrow 0$ along this subsequence. This contradicts $\lim _{\sigma \downarrow 0} z^{*}=g>0$. Hence necessarily $\lim _{\sigma \downarrow 0} q_{g}=0$.

Theorem 3 reveals that whenever an equilibrium exists in which the good seller type mixes, this equilibrium converges to the pooling on $m=0$ equilibrium when the noise becomes small. The other equilibrium in which the bad type mixes converges to a mixed equilibrium that is insufficiently revealing; the bad type chooses $m=b$ with probability $q_{0}$ and $m=0$ otherwise, while the good type chooses $m=b$ for sure. Upon receiving message $b$ the buyer then decides to buy. Interestingly, Theorems 2 and 3 together reveal that for a very favorable prior, only pooling on $m=0$ can occur. Some amount of noise thus precludes informative signaling altogether in this case.

\section{References}

[1] Aoyagi, Masaki, and Guillaume R. Fréchette, 2006: "Collusion as Public Monitoring Becomes Noisy: Experimental Evidence", Discussion Paper, New York University.

[2] Bagwell, Kyle, 1995: "Commitment and Observability in Games". Games and Economic Behavior, 8, 271-280.

[3] Blume, Andreas, Oliver Board and Kohei Kawamura, 2007: "Noisy Talk", Theoretical Economics, forthcoming.

[4] Brandts, Jordi, and Charles A. Holt, 1992: "An Experimental Test of Equilibrium Dominance in Signaling Games", American Economic Review, 82, 1350-1365.

[5] Calveras, Aleix, 2003: "Closure Policy when Bank Inspection Can Be Manipulated", European Finance Review, 7, 385-408.

[6] Carlsson, Hans and Sudipto Dasgupta, 1997: "Noise-Proof Equilibria in TwoAction Signaling Games", Journal of Economic Theory, 77, 432-460.

[7] Chagnon, Napoleon A., 1988: "Life Histories, Blood Revenge, and Warfare in a Tribal Population", Science, 239, 985-992.

[8] Chagnon, Napoleon A., 1992: "Yanomamö - The Last Days of Eden", Harcourt Brace Jovanovich College Publishers. 
[9] Cho, In-Koo and David M. Kreps, 1987: "Signaling Games and Stable Equilibria", Quarterly Journal of Economics, 102, 179-221.

[10] Cooper, David, Susan Garvin and John Kagel, 1997a: "Signalling and Adaptive Learning in an Entry Limit Pricing Game", RAND Journal of Economics, 28, 662-683.

[11] Cooper, David, Susan Garvin and John Kagel, 1997b: "Adaptive Learning vs. Equilibrium Refinements in an Entry Limit Pricing Game", Economic Journal, 107, 553-575.

[12] Crawford, Vincent P. and Joel Sobel, 1982: "Strategic Information Transmission", Econometrica, 50, 1431-1451.

[13] Feltovich, Nick, Richmond Harbaugh and Ted To, 2002: "Too Cool for School? Signalling and Countersignalling", RAND Journal of Economics, 33, 630-649.

[14] Güth, Werner, Wieland Müller and Yossi Spiegel, 2006: "Noisy Leadership: An Experimental Approach", Games and Economic Behavior, 57, 37-62.

[15] Hertzendorf, Mark N., 1993: "I'm Not a High-Quality Firm - But I Play One on TV", RAND Journal of Economics, 24, 236-247.

[16] Kanodia, Chadra, Rajdeep Singh and Andrew E. Spero, 2005: "Imprecision in Accounting Measurement: Can it be Value Enhancing?", Journal of Accounting Research, 43, 487-519.

[17] Kihlstrom, Richard E. and Michael H. Riordan, 1984: "Advertising as a Signal", Journal of Political Economy, 92, 427-450.

[18] Landeras, Pedro and J.M. Pérez de Villarreal, 2005: "A Noisy Screening Model of Education", Labour 19, 35-54.

[19] Maggi, Giovanni, 1999: "The Value of Commitment with Imperfect Observability and Private Information", RAND Journal of Economics, 30, 555574 .

[20] Matthews, Steven A. and Leonard J. Mirman, 1983: "Equilibrium Limit Pricing: The Effects of Private Information and Stochastic Demand", Econometrica, 53, 981-996.

[21] Milgrom, Paul R., 1981: "Good News and Bad News: Representation Theorems and Applications", The Bell Journal of Economics, 12, 380-391.

[22] Milgrom, Paul R., and D. John Roberts, 1986: "Price and Advertising Signals of Product Quality", Journal of Political Economy, 94, 796-821. 
[23] Miller, Ross. M., and Charles. R. Plott, 1985: "Product Quality Signaling in Experimental Markets", Econometrica, 53, 837-872.

[24] Nelson, Phillip, 1970: "Information and Consumer Behavior", Journal of Political Economy, 78, 311-329.

[25] Nelson, Phillip, 1974: "Advertising as Information", Journal of Political Economy, 82, 729-754.

[26] Spence, Michael, 1973: "Job Market Signaling", Quarterly Journal of Economics, 87, 355-374.

[27] van Damme, Eric and Sjaak Hurkens, 1997: "Games with Imperfectly Observable Commitment", Games and Economic Behavior, 21, 282-308. 
Table 1: Payoffs of seller and buyer over action-state pairs

\begin{tabular}{ccc}
\hline \hline & Seller is of bad type $(1-p)$ & Seller is of good type $(p)$ \\
\hline Buy & $b-m,-y$ & $g-m, x$ \\
Refrain & $-m, 0$ & $-m, 0$ \\
\hline \hline
\end{tabular}

Remarks: The first (second) number in each cell refers to the seller's (buyer's) payoffs. $g>b>0$ and $x, y>0$ are parameters of the model. $m \geq 0$ denotes the signal costs chosen by the seller.

Table 2: Overview of separating equilibria

\begin{tabular}{ccccccccc}
\hline \hline \multicolumn{8}{c}{ Amount of noise $\sigma$} \\
\cline { 2 - 9 } & $\leq 20$ & 25 & 40 & 75 & 100 & 120 & 140 & $\geq 145$ \\
\hline$z_{h}^{*}$ & - & 53.14 & 75.66 & 114.26 & 132.12 & 141.02 & 145 & - \\
$m_{g}$ & - & 101.28 & 142.21 & 206.43 & 228.80 & 231.62 & 216.63 & - \\
\hline \hline
\end{tabular}

Remarks: This table is based on the parameter values used in the experiment: $p=0.5, g$ $=400, b=90, x=300$ and $y=450$ (so $\beta^{*}=0.6$ ). In the experiment we only consider four different values of $\sigma$, viz. $0,10,40$ and 120. A dash implies that a separating equilibrium does not exist. 
Table 3: Overview of mixed strategy equilibria

\begin{tabular}{cccccccccc}
\hline \hline & \multicolumn{4}{c}{ Bad type mixes } & & \multicolumn{3}{c}{ Good type mixes } \\
\cline { 2 - 4 } \cline { 7 - 9 }$\sigma$ & $z_{h}^{*}$ & $q_{b}$ & $m_{b}$ & $m_{g}$ & & $z_{h}^{*}$ & $q_{g}$ & $m_{g}$ \\
\hline 1 & 88.98 & 0.15 & 89.66 & 90.16 & & 396.53 & $\approx 0$ & 399.71 \\
5 & 77.95 & 0.15 & 87.88 & 91.11 & & 385.14 & $\approx 0$ & 398.30 \\
10 & 69.06 & 0.15 & 85.05 & 92.59 & & 372.74 & $\approx 0$ & 396.28 \\
40 & - & - & - & - & & 314.21 & $\approx 0$ & 380.75 \\
120 & - & - & - & - & & 200.35 & 0.25 & 290.95 \\
\hline \hline
\end{tabular}

Remarks: This table is based on the parameter values used in the experiment: $g=400, b$ $=90, x=300, y=450$ and $p=0.5$. In the experiment we only consider the four different values of $\sigma$ of $0,10,40$ and 120. A dash implies that a mixed strategy equilibrium does not exist for the given value of $\sigma$.

Table 4: Experimental design

\begin{tabular}{cccc}
\hline \hline Treatment & $\sigma$ & $\begin{array}{c}\text { \# of } \\
\text { matching } \\
\text { groups }\end{array}$ & $\begin{array}{c}\text { \#subjects per } \\
\text { matching group }\end{array}$ \\
\hline$\sigma_{0}$ & 0 & 4 & 8 \\
$\sigma_{10}$ & 10 & 5 & 8 \\
$\sigma_{40}$ & 40 & 6 & 8 \\
$\sigma_{120}$ & 120 & 8 & 8 \\
\hline \hline
\end{tabular}

Remarks: Per period payoffs for the subjects are given in Table 1, with $b=90, g=400, x=300, y=450$ and $p=0.5$. 
Table 5: Pooling and separating signal costs (SC) in periods 21-40

\begin{tabular}{|c|c|c|c|c|c|c|c|}
\hline & \multicolumn{3}{|c|}{ Pooling: SC $<90$} & \multicolumn{3}{|c|}{ Separating: SC $\geq 90$} & \multirow{2}{*}{$\begin{array}{c}\text { Predicted } \\
\text { separating eq. }\end{array}$} \\
\hline & $\mathrm{n}$ & Actual & Emp. best response & $\mathrm{n}$ & Actual & Emp. best response & \\
\hline \multicolumn{8}{|c|}{ Low type } \\
\hline$\sigma_{0}$ & 149 & $0.7(7.0)$ & $2.1(13.0)$ & 11 & $112.3(11.7)$ & $0.0(0.0)$ & 0.0 \\
\hline$\sigma_{10}$ & 200 & $2.3(12.9)$ & $0.0(0.0)$ & - & - & - & 0.0 \\
\hline$\sigma_{40}$ & 235 & $2.8(11.7)$ & $0.0(0.0)$ & 5 & $124.0(28.8)$ & $0.0(0.0)$ & 0.0 \\
\hline$\sigma_{120}$ & 319 & $1.6(7.0)$ & $0.0(0.0)$ & 1 & $110.0(0.0)$ & $0.0(0.0)$ & 0.0 \\
\hline \multicolumn{8}{|c|}{ High type } \\
\hline$\sigma_{0}$ & 1 & $80.0(0.0)$ & $80.0(0.0)$ & 159 & $97.9(11.4)$ & $93.8(4.4)$ & 90.0 \\
\hline$\sigma_{10}$ & 4 & $70.0(4.1)$ & $112.5(15.0)$ & 196 & $113.6(15.9)$ & $117.9(11.6)$ & $48.7^{*}$ \\
\hline$\sigma_{40}$ & 18 & $38.3(33.7)$ & $149.6(16.2)$ & 222 & $140.5(41.5)$ & $156.3(17.5)$ & 142.2 \\
\hline$\sigma_{120}$ & 187 & 17.7 (29.9) & 190.8 (73.8) & 133 & $158.3(55.5)$ & $187.4(62.5)$ & 231.6 \\
\hline \multicolumn{8}{|c|}{ Ranksum tests high type (p values) } \\
\hline$\sigma_{0} \mathrm{v}$ & & & & & 0.09 & & \\
\hline$\sigma_{0} \mathrm{v}$ & & & & & 0.01 & & \\
\hline$\sigma_{0} \mathrm{Vs}$ & & & & & 0.01 & & \\
\hline$\sigma_{10} \mathrm{~V}$ & & & & & 0.10 & & \\
\hline$\sigma_{10} \mathrm{~V}$ & & & & & 0.11 & & \\
\hline$\sigma_{40} \mathrm{~V}$ & & & & & 0.90 & & \\
\hline
\end{tabular}

Remarks: $\mathrm{n}$ gives the number of observations. Standard deviations (based on individual observations) in parentheses. * When $\sigma=10$ only pooling on zero is a Nash equilibrium; a choice for 48.7 by the high type is a best response given that the low type chooses a signal cost of zero (but not vice versa). Between treatment comparisons are based on Mann-Whitney ranksum tests (performed at the group level). 
Table 6: Average actual outcomes per group and comparison with best response (periods 21-40)

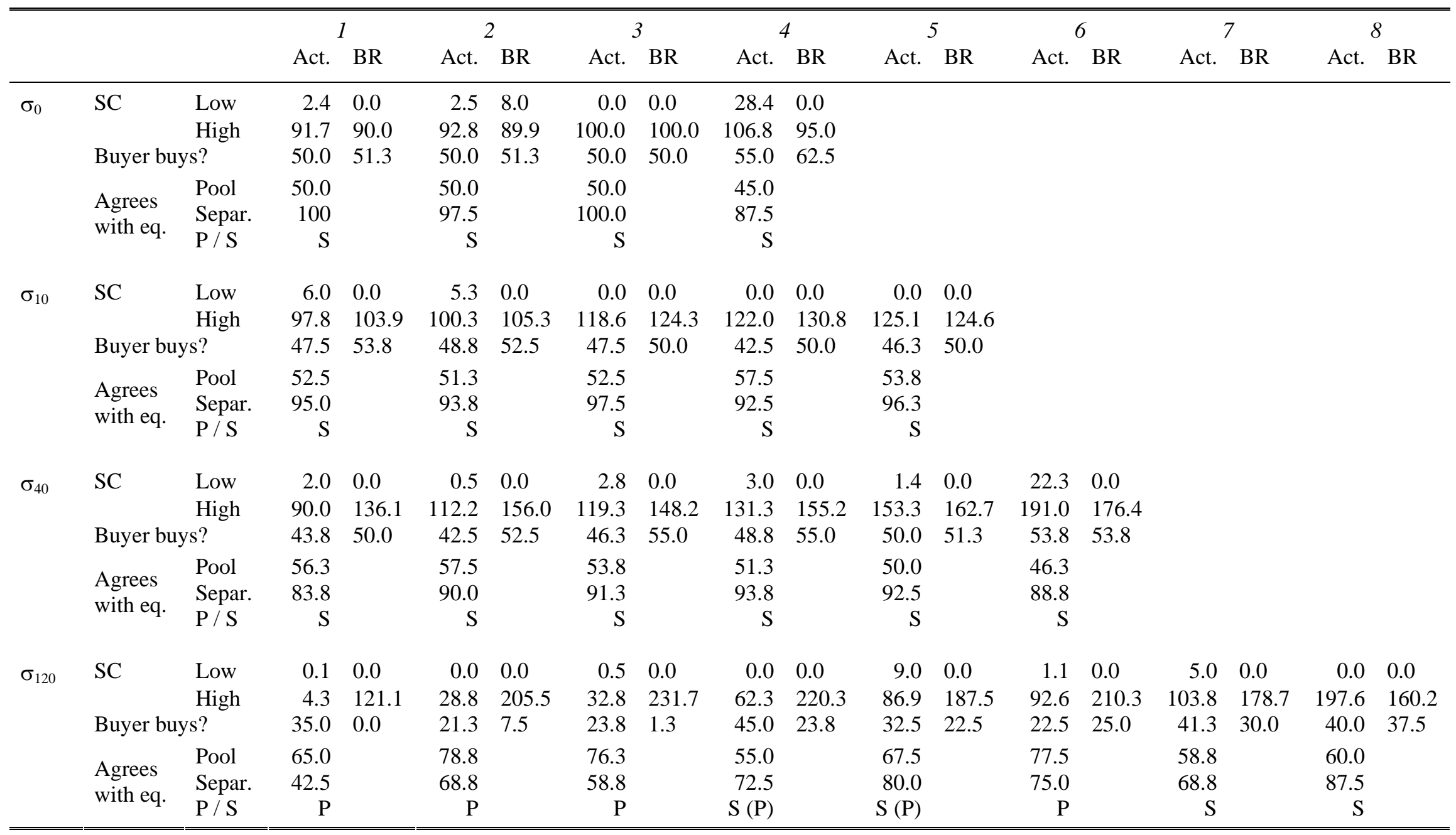

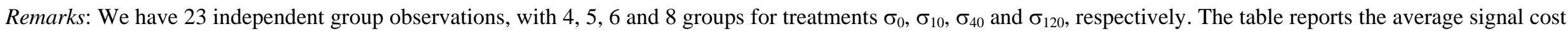

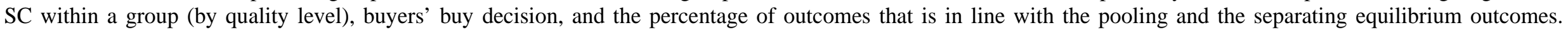

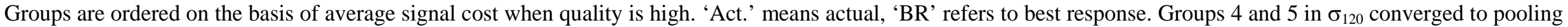
with the strategy method: in periods $41-50,87.5 \%$ (75.0\%) and 77.5\% (75.0\%) of the outcomes agreed with pooling (separating) in groups 4 and 5 , respectively. 
Table 7: Average estimated cutoff levels and tests for equality (periods 21-40)

\begin{tabular}{|c|c|c|c|c|c|c|}
\hline & & Pooling group & & & Separating grou & \\
\hline & $\begin{array}{l}\text { Estimated } \\
\text { actual } \\
\text { (stan. Dev.) }\end{array}$ & $\begin{array}{c}\text { Predictions } \\
\text { best response } \\
\text { [equilibrium] }\end{array}$ & $\begin{array}{c}\text { Actual vs. } \\
\text { best response } \\
\text { [equilibrium] }\end{array}$ & $\begin{array}{l}\text { Estimated } \\
\text { actual } \\
\text { (stan. dev.) }\end{array}$ & $\begin{array}{c}\text { Predictions } \\
\text { best response } \\
\text { [equilibrium] }\end{array}$ & $\begin{array}{c}\text { Actual vs. } \\
\text { best response } \\
\text { [equilibrium] }\end{array}$ \\
\hline$\sigma_{0}$ & - & - & - & 94.0 & 53.0 & 0.07 \\
\hline & & & & (4.1) & [90.0] & {$[0.11]$} \\
\hline$\sigma_{10}$ & - & - & - & 96.2 & 57.8 & 0.04 \\
\hline & & & & (15.1) & {$\left[25.6^{*}\right]$} & {$[0.04]$} \\
\hline$\sigma_{40}$ & - & - & - & 98.5 & 75.3 & 0.03 \\
\hline & & & & (22.4) & [75.7] & [0.03] \\
\hline$\sigma_{120}$ & 211.5 & 330.9 & 0.72 & 96.9 & 122.2 & 0.47 \\
\hline & $(166.2)$ & [800] & {$[0.07]$} & $(84.8)$ & [141.0] & {$[0.07]$} \\
\hline Rank & um tests (p-va & ues) & & & & \\
\hline$\sigma_{0} \mathrm{Vs}$ & & & & 0.62 & & \\
\hline$\sigma_{0} \mathrm{vs}$ & & & & 0.39 & & \\
\hline$\sigma_{0} \mathrm{vs}$ & $\sigma_{120}$ & & & 0.25 & & \\
\hline$\sigma_{10} \mathrm{~V}$ & $\sigma_{40}$ & & & 1.00 & & \\
\hline$\sigma_{10} \mathrm{~V}$ & $\sigma_{120}$ & & & 0.22 & & \\
\hline$\sigma_{40} \mathrm{~V}$ & $\sigma_{120}$ & & & 0.34 & & \\
\hline
\end{tabular}

Remarks: For each buyer the actual cutoff level was estimated on the basis of the choices in periods 21-40. The cutoff level was set such that the number of errors against the cutoff level was minimized. Standard deviations (based on observations per person) in parentheses; equilibrium predictions/comparisons appear in square brackets. (* When $\sigma=10$ only pooling on zero is a Nash equilibrium.) Within treatment comparisons are based on Wilcoxon tests performed at the matching group level, between treatment comparisons are based on Mann-Whitney tests performed at the matching group level. 
Table 8: Consistency best / behavioral response with actual buy decisions (periods 21-40)

\begin{tabular}{|c|c|c|c|c|c|}
\hline & \multirow[t]{2}{*}{ Buy? } & \multicolumn{2}{|c|}{ Model: best response } & \multicolumn{2}{|c|}{ Model: behavioral response } \\
\hline & & No & Yes & No & Yes \\
\hline \multirow[t]{2}{*}{$\sigma_{0}$} & No & 148 & 8 & 148 & 8 \\
\hline & Yes & - & 164 & - & 164 \\
\hline \multirow[t]{2}{*}{$\sigma_{10}$} & No & 194 & 20 & 206 & 8 \\
\hline & Yes & 1 & 185 & 7 & 179 \\
\hline \multirow[t]{2}{*}{$\sigma_{40}$} & No & 218 & 34 & 235 & 17 \\
\hline & Yes & 8 & 220 & 16 & 212 \\
\hline \multirow[t]{2}{*}{$\sigma_{120}$} & No & 414 & 17 & 386 & 45 \\
\hline & Yes & 108 & 101 & 77 & 132 \\
\hline \multirow[t]{2}{*}{$\sigma_{\text {all }}$} & No & 974 & 79 & 975 & 78 \\
\hline & Yes & 117 & 670 & 100 & 687 \\
\hline
\end{tabular}

Remarks: The cells list the number of cases that fall into the specific row (actual buy decision) and the corresponding column (model prediction). $\sigma_{\text {all }}$ collapses all cases of all treatments. 
Table 9: Signal costs sellers, best responses and behavioral responses (periods 21-40)

\begin{tabular}{|c|c|c|c|c|c|c|}
\hline & Quality & Actual & $\begin{array}{c}\text { Best } \\
\text { response }\end{array}$ & $\begin{array}{c}\text { Behavioral } \\
\text { response }\end{array}$ & | Best-Actual | & | Behav.-Actual | \\
\hline \multirow[t]{2}{*}{$\sigma_{0}$} & Low & $8.3(29.3)$ & $2.0(12.5)$ & $0.2(1.8)$ & $10.3(31.3)$ & 8.5 (29.3) \\
\hline & High & $97.8(11.5)$ & 93.7 (4.5) & $94.3(4.0)$ & $4.1(10.8)$ & 3.9 (10.7) \\
\hline \multirow[t]{2}{*}{$\sigma_{10}$} & Low & $2.3(12.9)$ & $0.0(0.0)$ & $0.4(3.1)$ & $2.3(12.9)$ & $2.3(12.1)$ \\
\hline & High & $112.8(16.9)$ & $117.8(11.7)$ & $111.1(10.5)$ & $10.1(10.1)$ & $8.2(10.7)$ \\
\hline \multirow[t]{2}{*}{$\sigma_{40}$} & Low & $5.3(21.2)$ & $0.0(0.0)$ & $3.3(7.6)$ & $5.3(21.2)$ & $7.9(21.0)$ \\
\hline & High & $132.8(49.0)$ & $155.8(17.4)$ & $128.4(18.8)$ & $36.8(33.0)$ & $24.1(34.8)$ \\
\hline \multirow[t]{2}{*}{$\sigma_{120}$} & Low & $2.0(9.2)$ & $0.0(0.0)$ & $2.0(4.4)$ & $2.0(9.2)$ & $3.2(9.0)$ \\
\hline & High & 76.1 (81.3) & $189.4(69.2)$ & 15.7 (43.0) & $131.6(83.8)$ & $71.0(77.1)$ \\
\hline \multirow[t]{2}{*}{$\sigma_{\text {all }}$} & Low & 4.0 (18.3) & $0.3(5.3)$ & $1.7(5.1)$ & $4.4(19.0)$ & $5.2(18.2)$ \\
\hline & High & $102.6(59.3)$ & $148.4(55.7)$ & 79.5 (55.3) & $58.3(76.0)$ & 33.4 (56.8) \\
\hline
\end{tabular}

Remarks: Standard deviations (based on individual observations) in parentheses. $\sigma_{\text {all }}$ collapses all cases of all treatments. The last two columns give the absolute differences between actual signal costs and best responses and behavioral responses, respectively. 
Table 10: Seller profits conditional on signal and type (periods 21-40)

\begin{tabular}{|c|c|c|c|c|}
\hline & \multicolumn{2}{|c|}{ Low type } & \multicolumn{2}{|c|}{ High type } \\
\hline & Pooling & Separating & Pooling & Separating \\
\hline & $\mathrm{SC}<90$ & $\mathrm{SC} \geq 90$ & $\mathrm{SC}<90$ & $\mathrm{SC} \geq 90$ \\
\hline \multirow[t]{2}{*}{$\sigma_{0}$} & -0.7 & -46.8 & -80.0 & 294.5 \\
\hline & $(7.0)$ & (46.3) & $(0.0)$ & $(60.2)$ \\
\hline \multirow[t]{2}{*}{$\sigma_{10}$} & -0.9 & - & 30.0 & 257.8 \\
\hline & (9.6) & & (200.0) & (100.6) \\
\hline \multirow[t]{2}{*}{$\sigma_{40}$} & 3.3 & -52.0 & 117.2 & 221.7 \\
\hline & $(24.6)$ & (37.0) & (206.3) & (119.1) \\
\hline \multirow[t]{2}{*}{$\sigma_{120}$} & 22.6 & -110 & 80.7 & 73.3 \\
\hline & $(40.4)$ & $(0.0)$ & (170.2) & (189.8) \\
\hline
\end{tabular}

Remarks: The cells list average profits. Standard deviations (based on individual observations) in parentheses. 
Figure 1a: signal densities and posterior beliefs in the no noise case

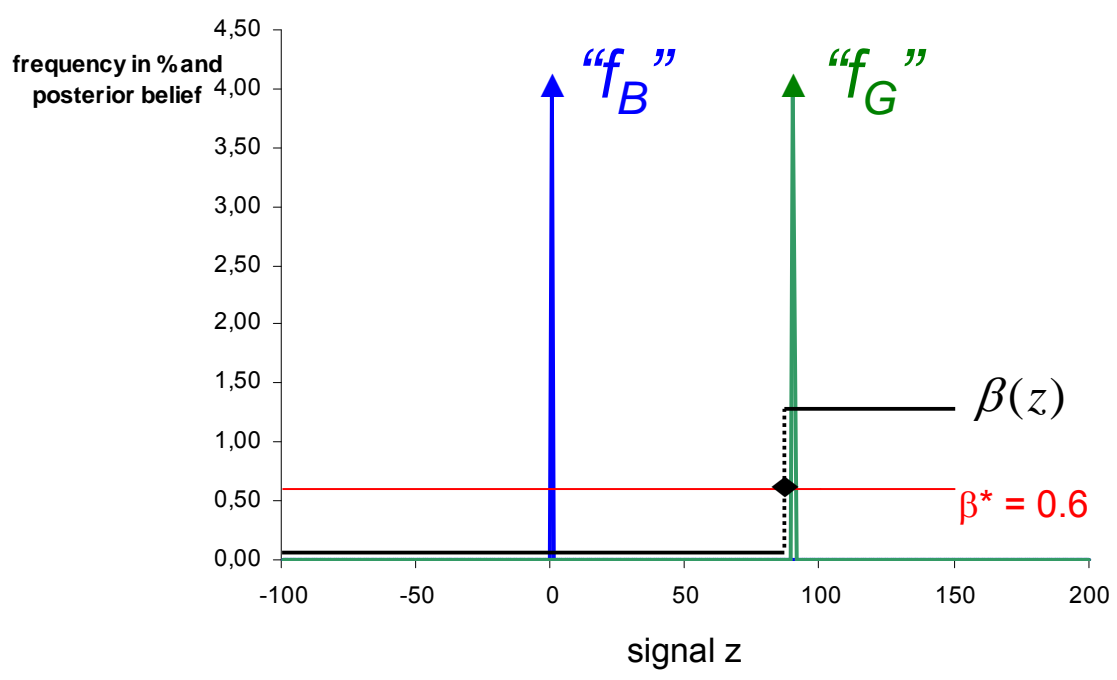

Figure $1 \mathrm{~b}$ : signal densities and posterior beliefs in the $\sigma=10$ case

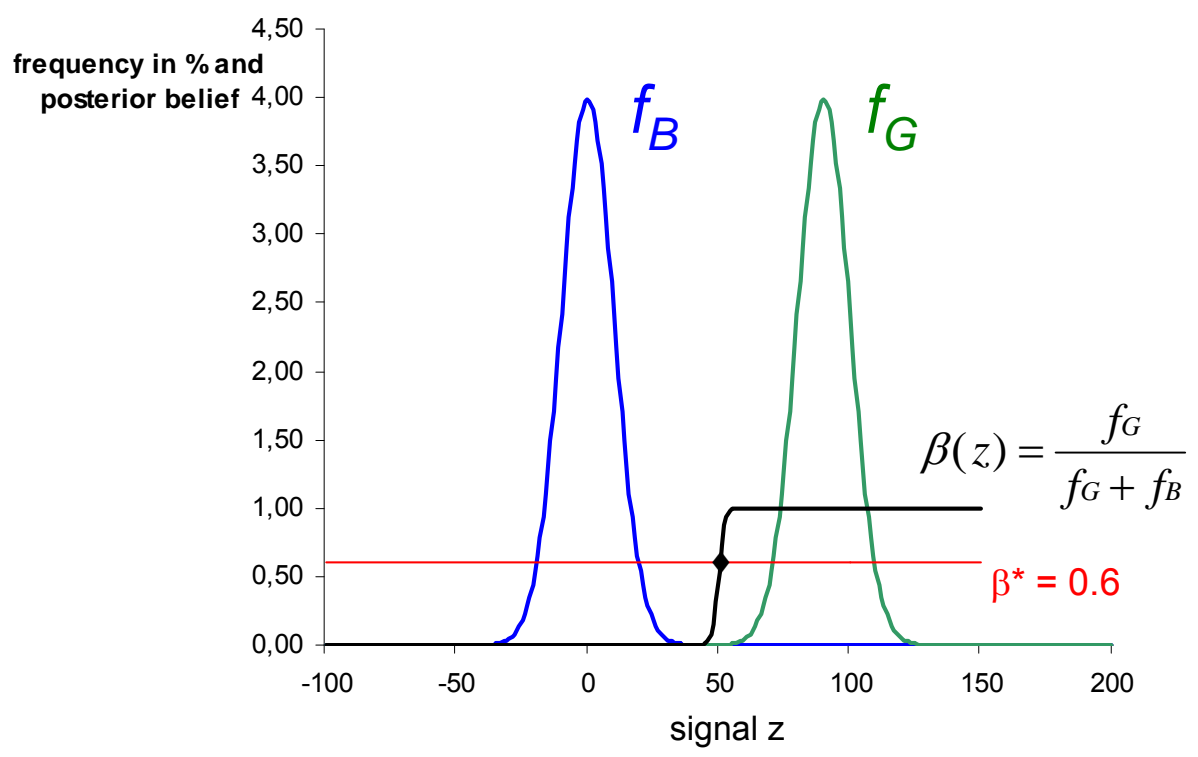


Figure 2a: Social history window buyers (example).

\begin{tabular}{|c|c|c|c|c|}
\hline row number & signal & quality & signal cost & buyer buys? \\
\hline 1 & 142 & HIGH & 146 & YES \\
\hline 2 & 142 & LOW & 118 & YES \\
\hline 3 & 98 & HIGH & 101 & NO \\
\hline 4 & -2 & LOW & 5 & NO \\
\hline & & & & \\
\hline & & & & \\
\hline & & & & \\
\hline
\end{tabular}

Figure 2b: Social history window sellers (example).

\begin{tabular}{|c|c|c|c|c|}
\hline row number & signal cost & signal & buyer buys? & quality \\
\hline 1 & 142 & 146 & YES & HIGH \\
\hline 2 & 142 & 118 & YES & HIGH \\
\hline 3 & 98 & 101 & NO & LOW \\
\hline 4 & 5 & -2 & NO & LOW \\
\hline & & & & \\
\hline & & & & \\
\hline & & & & \\
\hline
\end{tabular}


Figures 3a-3h: running histograms for periods 21-40; for each signal cost the relative frequency of cases in the interval [signal cost-5, signal cost+5] is displayed.

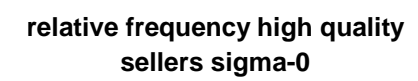
sellers sigma-0

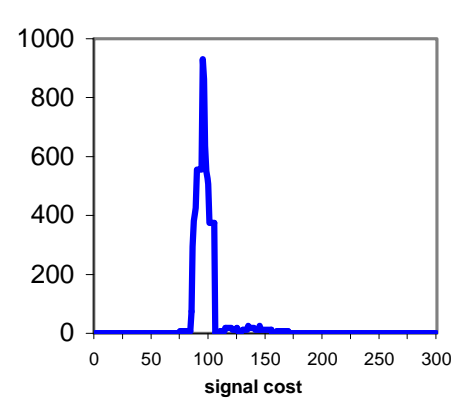

\section{relative frequency low quality sellers sigma-0}

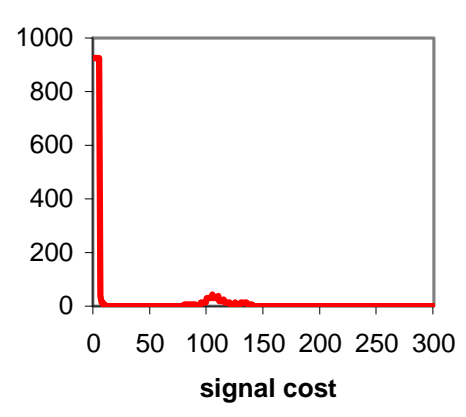

relative frequency high quality sellers sigma-10

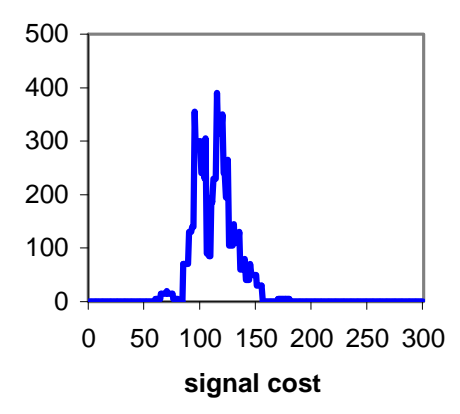

relative frequency low quality sellers sigma-10

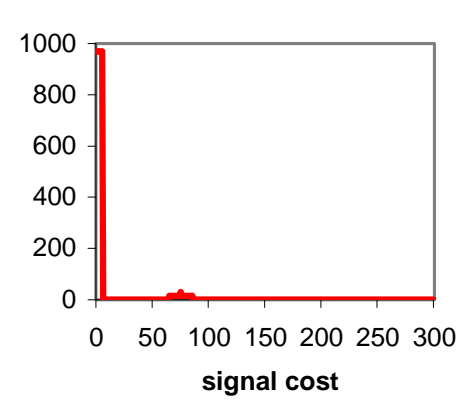

relative frequency high quality sellers sigma-40

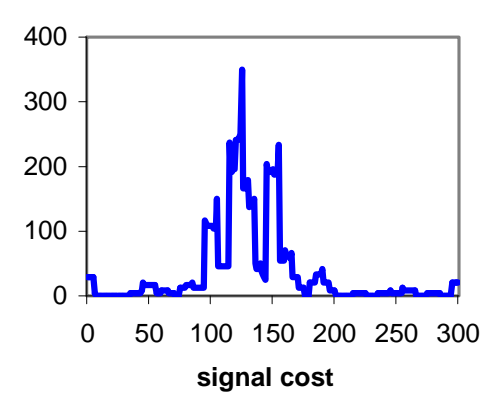

relative frequency low quality sellers sigma-40

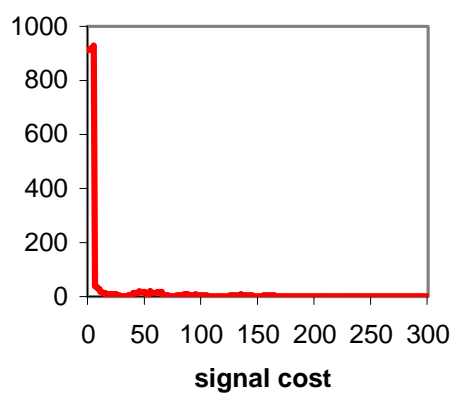

relative frequency high quality sellers sigma-120

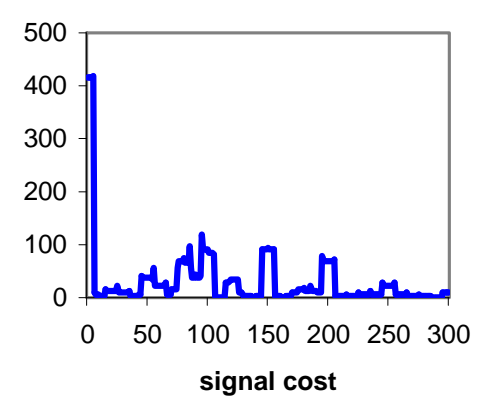

relative frequency low quality sellers sigma-120

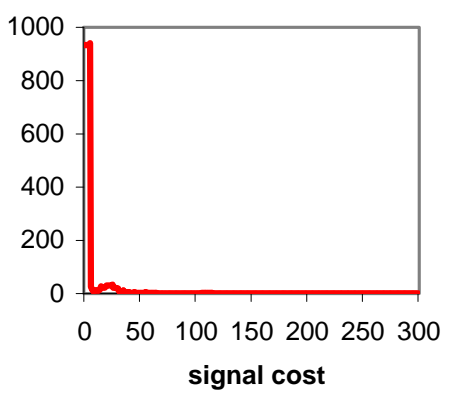


Figures 4a-4d: expected profit buyer as function of cutoff given actual behavior of sellers in periods $21-40$.
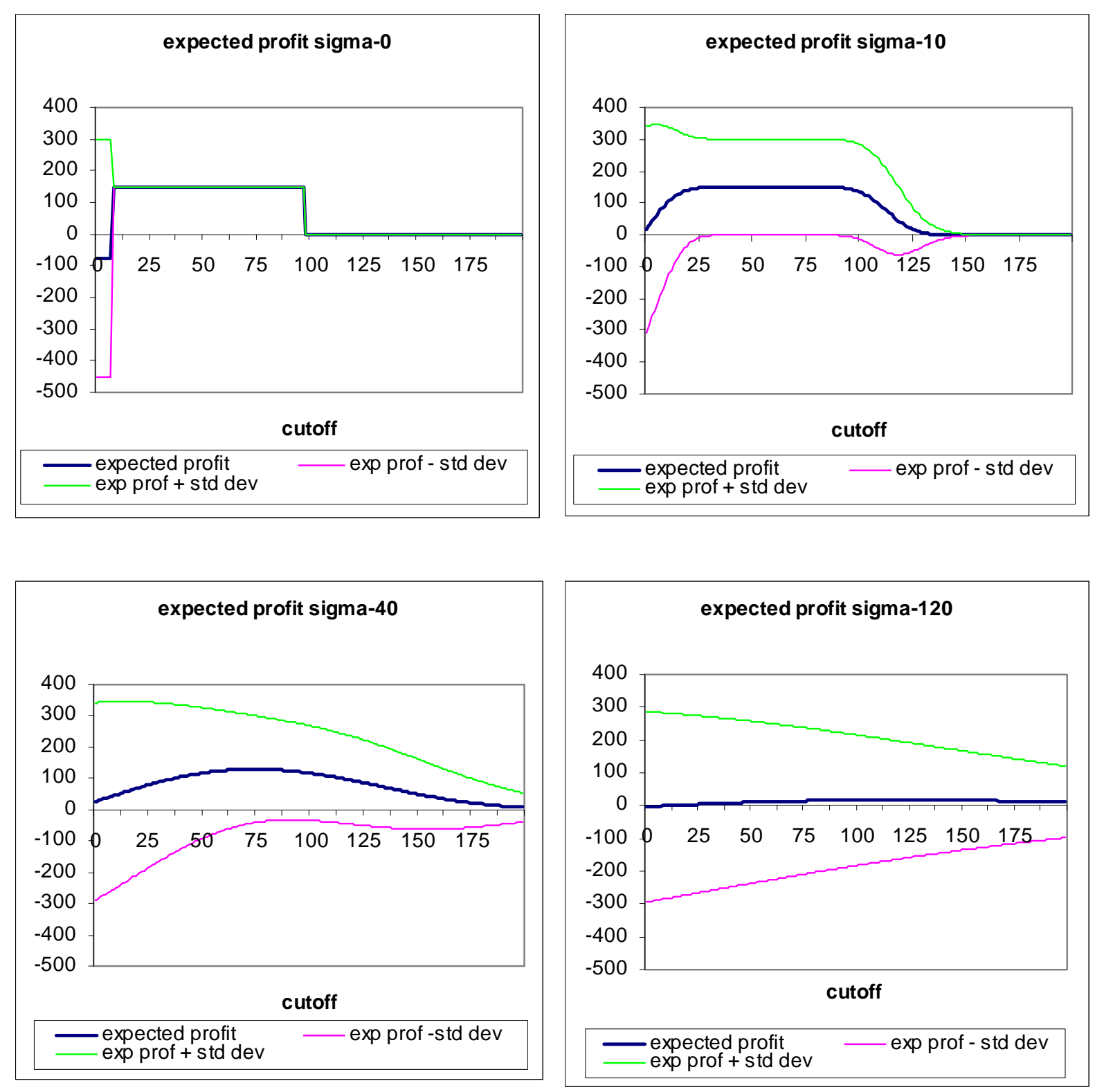
Figures 5a-5d: expected profit high-quality seller as function of signal cost given actual behavior of buyers in periods 21-40.
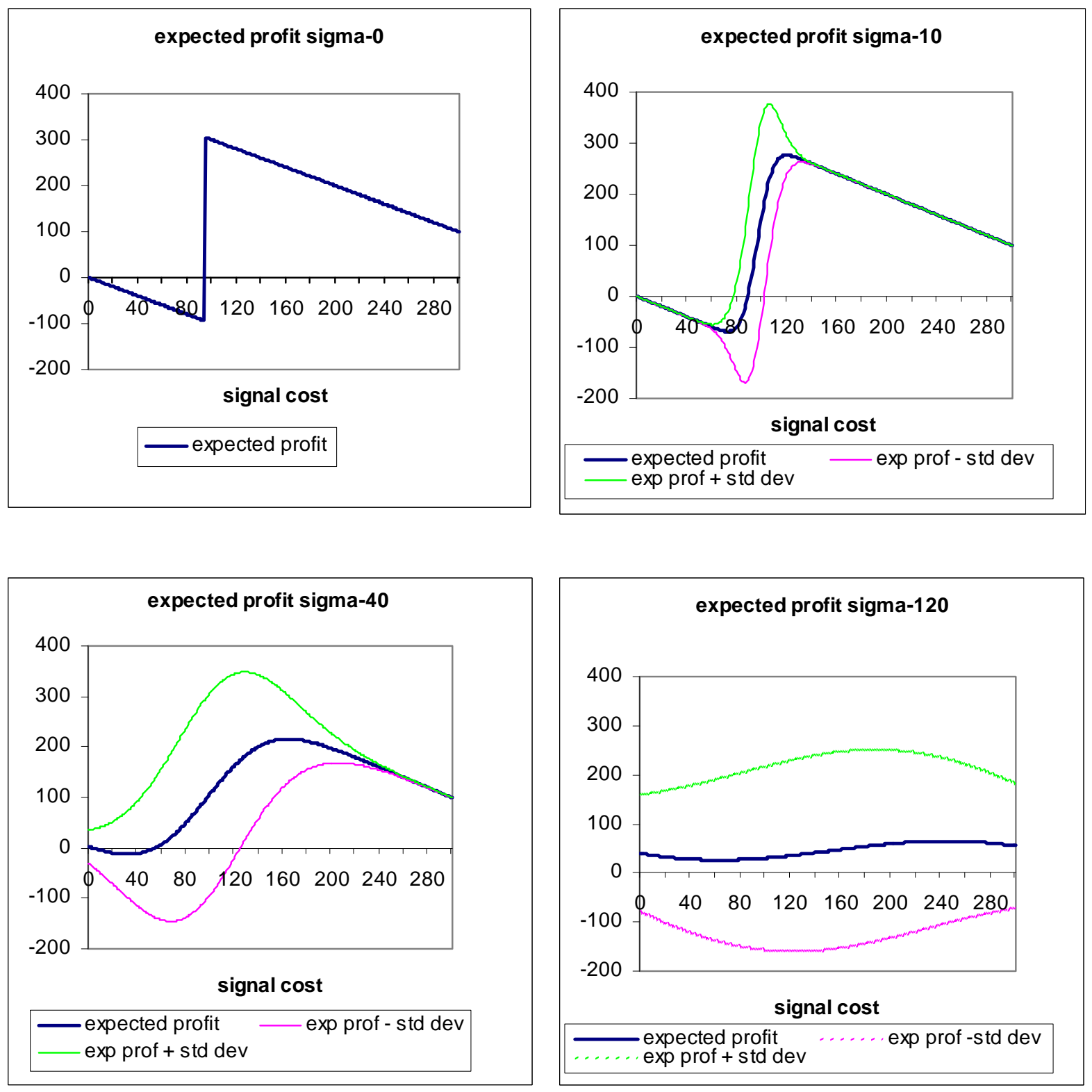